\title{
Modified PDMS packaging of sensory e-textile circuit microsystems for improved robustness with washing
}

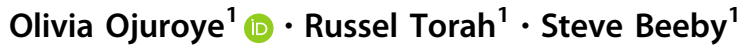

Received: 17 August 2018 / Accepted: 29 April 2019/Published online: 18 May 2019

(C) The Author(s) 2019

\begin{abstract}
Electronic Textiles (e-textiles) should ideally be handled and cleaned like traditional textiles. Therefore, we can expect e-textiles to be machine washed or hand washed. As e-textiles enhance traditional fabrics with electronic functionality, any embedded microsystem i.e., flexible electronic circuits, will be expected to survive and show functionality after the e-textile has been washed multiple times to ensure the garment is practical. Therefore, the choice of encapsulation material for microsystems in a textile must be hydrophobic and offer minimal expansion when washed and ensure the electronics are undetectable when the textile is handled or cleaned. This paper evaluates five different base/curing agent mixing ratios-5:1, 7:1, 10:1, 15:1, and 20:1—of commercial polydimethylsiloxane (PDMS) as an electronic packaging encapsulation. Contact angle and aqueous permeability experiments were conducted to tailor the PDMS mixture specifically for washable e-textile applications. The experimental results show that 20:1 PDMS is the most suitable as it is sufficiently hydrophobic with minimal swelling in commercial washing machine trials. Following this, a $40.3 \mu \mathrm{m}$-thick 20:1 conformal encapsulation of PDMS upon an touch and proximity flexible circuit that can be integrated into textiles via knitting and/or weaving, was examined. Results show the washing spin speed is a crucial factor with washing cycle duration having minimal impact when determining circuit functionality survival. Overall, the e-textiles in this work survived between 10 and 15 washes with microscopic inspection of the circuits revealing failure of the external wires but not the PDMS encapsulation—suggesting its sufficient robustness and durability as a suitable encapsulation material for washable electronic textiles.
\end{abstract}

\section{Introduction}

Polydimethylsiloxane (PDMS) is an organic polymer commonly used in the bioscience field for microfluidics (Fujii 2002) and in situ microelectronics (Mark and Pan 1982). It is used for its chemical inertness, excellent biocompatibility, broad range of mechanical properties, and it is non-flammable. It is fabricated as a two-part silicone elastomer where its chemical and mechanical properties are altered via the mixing ratio of base agent to curing agent. Sylgard 184 Silicone Elastomer, from Dow Corning,

Olivia Ojuroye

oo2g12@ecs.soton.ac.uk

Russel Torah

rnt@ecs.soton.ac.uk

Steve Beeby

spb@ecs.soton.ac.uk

1 Electronics and Computer Science, University of Southampton, Southampton, UK recommends a 10:1 (base to curing agent) mixing ratio to be well-suited to electrical/electronic applications (Jeong et al. 2015). It is the most commonly used mixing ratio in electronics and bioelectronic applications (Jeong et al. 2015). PDMS' empircal formula is (C2H6OSi)n, where $n$ is the number of repeating $(\mathrm{OSi}(\mathrm{CH} 3) 2)$ monomoers (Fig. 1). Changing the cross-linking density of PDMS (low $\mathrm{n}$ for near-liquid PDMS and high $\mathrm{n}$ for semi-solid PDMS) changes its chemical structure can control the extent of PDMS' surface hydrophobicity (Armani et al. 1999). Literature reports microsystems integrated into textiles but not packaging the entire system for hydrophobic purposes to protect it from water, detergent, and fabric conditioner solutions. Instead, only MEMS and silicon chips are glob-topped in rigid resin and not the entire circuit (Cherenack et al. 2010) to allow the final textile to flex. Some electronics and microsystems are external to the textile due to large circuit size and rigidity (Zysset et al. 2012) that are not compatible with textile construction machinery, and the packaging substrates used 


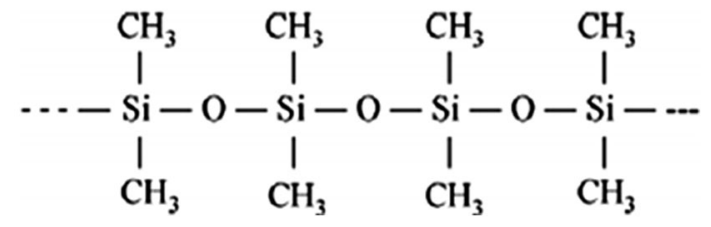

Fig. 1 Chemical molecular structure of polydimethylsiloxane (PDMS) from Morent et al. 2007)

are at times inflexible. Hence, they offer insufficient encapsulation to protect the electronics from water, solvents, and flammable environments-and not suitable for flexible circuits integrated into textiles.

PDMS is porous, making the bulk polymer permeable to different micromolecules found in solvents (Toepke and Beebe 2006). PDMS swelling can occur due to aqueous solutions or gaseous permeating the substrate and/or chemical attacks of organic alkaline or acidic solvents (van Dam 2006) which could be the case during washing conditions. By changing the PDMS fabrication, it is possible to select the mixing ratio to offer best waterproof and swelllimiting properties (Fig. 2).

For consumer, wearable or non-wearable textiles, a basic requirement is their ability to be cleaned. If electronic textiles (e-textiles,) formed by electronic circuits integrated into textiles at yarn-level are to be considered functional, safe, and durable for use they need to survive a sufficient number of washing cycles to be considered robust (Wainwright 2016). It would be added maintenance for consumers to remove all electronic components from an e-textile before washing. As a result, research has focused on encapsulating electronic components in aqueous-resistant substrates. Such, that the electronics can be washed and survive the washing cycle process. Recently, PDMS as an encapsulation for e-textile circuits has been investigated with the aim of improving washability. Work by Tao et al. (2018) report of a miniaturised, PDMS encapsulated, reliable, and fully washable Bluetooth activity monitoring system that can feature on wearable textiles. Other literature has reported washing cycle survival of less than 20 before failure (Linz et al. 2005; Merritt et al. 2009;

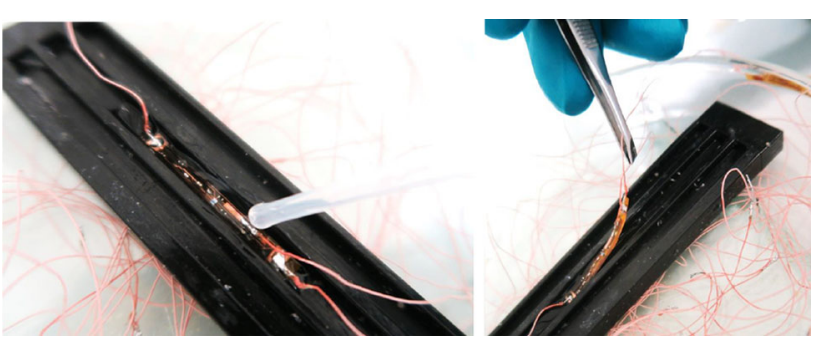

Fig. 2 Photos of applying PDMS via a pipette to cover circuit surface (left) then removal of the uncured PDMS-packaged circuit out of the 3D printed mould (right)
Varnaite and Katunskis 2009) which is insufficiently durable for most long-term usage. Linz et al. (2005) published results from an on-going $30{ }^{\circ} \mathrm{C}$ wash experiment where circuits had survived five washes without failing. The circuits would fail if its component globtop, circuit plastic moulded packaging or conductive textile embroidered wires disconnected during the wash. Merritt et al. (2009) reported its sensing electrodes also surviving five washing cycles; however its failure was measured by cracks in the electrode due to washing that would prevent it passing a sine-wave function test which would confirm its usability. Varnaite et al. (2009) conducted its washing test evaluating the change in conductivity of yarns and not circuity. It stated that yarns failed the washing test when its electrostatic properties became impaired. It focused on measuring the effect of washing after the fifth wash as this recorded the most distinctive increase in resistance.

Some literature also report washing tests of circuits for e-textile applications that are not truly representative of how washing of textiles occurs. For example, washing their e-textiles in separate wash bags (Rantanen et al. 2002), (Satharasinghe et al. 2018)—normally used for washing delicate clothing. Nonetheless, the inclusion of this protective bag is not representative of how the majority of apparel and furnishing textiles are treated in a washing machine. Furthermore, the wash bag is said to a contributing factor to minimise the mechanical stressess from agitation experienced by textiles during machine washing (Satharasinghe et al. 2018). This therefore presents the need to conduct washing tests on e-textiles that experience truly reflective mechanical agitations from the cyclic motions of a washing machine. Other literature report washing e-textiles which include detergent but not fabric conditioner (Mattmann et al. 2008; Fu et al. 2015) - and even washing with no other laundry in the machine $(\mathrm{Fu}$ et al. 2015). One of the greatest number of cycles recorded in literature for smart textile washing are 20 (Kazani et al. 2012) and 50 washing cycles (Kaappa et al. 2017). However, these washing tests are not a true comparison as they were not circuits integrated into a textile. Kazani et al. (2012) investigated printed conductive wires and their durability after washing, not electronic circuits, and Kaappa et al. (2017) measured the change in sensing ability of dry electrode pads and was not washed with a connected electronic circuit. Hence, there is a need for a truly representative washing test of e-textiles which can contribute towards new washing standards. Such an e-textile washing standard can be closely matched to traditional textile washing standards but the e-textile under test features a fully integrated and fully functional circuit.

Thus, this paper has explored the use of PDMS as a flexible, hydrophobic encapsulating layer upon flexible electronic sensors to be woven to form an e-textile. The 
experiment evaluated the e-textile's robustness through these washing tests, compliant with the British Standard BE ISO 6330:2012 standard for domestic washing. The intention of the washing test is to investigate PDMS as an e-textile circuit encapsulation-for example, when cleaned using a washing machine and/or handwashing with detergent and fabric conditioner. Investigating PDMS for this specific e-textile use-case would be fundamental its compatiblity for consumer usage. The motivation of this paper is to contribute to e-textile washing standards which have not yet been established. Additionally, to inform future e-textile consumers which washing machine settings to safely wash them for maintained robustness and prolonged functionality. This paper includes the initial PDMS mixing ratio experimental investigation on how a capacitive proximity and touch sensory circuit-with the experimentally-verified PDMS mixing ratio-was embedded into a textile to take part in a washing test.

\section{Methodology}

\subsection{Polydimethylsiloxane fabrication for experiments}

Sylgard 184 PDMS was mixed at 5:1, 7:1, 10:1, 15:1, and 20:1 base to curing agent ratios with $20 \mathrm{~g}$ of base agent for each mixture. Each was mixed in a beaker for $1 \mathrm{~min}$ and degassed in a vacuum chamber for $20 \mathrm{~min}$ to remove any bubbles. For the contact angle tests, each degassed mixture was poured into separate $8 \mathrm{~cm}$-diameter glass Petri dishes and cured at $140{ }^{\circ} \mathrm{C}$ for $10 \mathrm{~min}$. For the swelling tests, five strip-shaped PDMS per mixing ratio were made with the same fabrication method. They were cut into dimensions $5 \mathrm{~cm}(\mathrm{~L}) \times 0.3 \mathrm{~cm} \quad(\mathrm{~W}) \times 0.3 \mathrm{~cm}(\mathrm{H})$, similar in size to a flexible capacitive electronic sensory circuit currently under development by the author (Ojuroye et al. 2019). For the washing machine tests, the experimentally-verified PDMS mixing ratio was fabricated again using the same method. It was then deposited into a 3D-printed mould with $10 \mathrm{~cm}(\mathrm{~L}) \times 0.37 \mathrm{~cm}(\mathrm{~W})$ x 0.2 (H) dimensions using a pipette. The pipette tip was used to line the surface of the channel before tweezers were used to position a flexible touch and proximity sensing circuit on top of this PDMS layer inside the channel. The tweezers were used again to lay the flexible circuit flat to ensure the PDMS lined the bottom surface. Then, approximately $0.5 \mathrm{ml}$ of uncured PDMS mixture was dispensed via a pipette along the entire surface of the sensory circuit to encapsulate it. The PDMS mixture was self-leveling, ensuring that no gaps were present around the components and an even surface was achieved.
As part of a suspension method of applying a conformal coating of PDMS onto circuit, tweezers were then used to lift the circuit out of the now PDMS-filled channel and the circuit was suspended above the mould. The suspension was possible by putting parafilm tape over the copper wires soldered to each end of the circuit to compact them together, and using the 3D printed mould as a weight. The PDMS had a total self-leveling time of $5 \mathrm{~min}$ per circuit before entering the oven. All the circuits were suspended using the wires and thermally cured in the oven whilst suspended for $10 \mathrm{~min}$ at $140{ }^{\circ} \mathrm{C}$.

\subsection{Contact angle hydrophobicity experiment}

This research was funded in the UK by the Engineering and Physical Sciences Research Council (EPSRC) via the project: Novel manufacturing methods for Functional Electronic TexTiles (FETT). Grant number: EP/M015149/ 1.

These tests were performed using the DSA30 Krüss Contact Angle Machine (Fig. 3). Three aqueous washing environments were evaluated: distilled water, distilled water mixed with a commercial anionic-based detergent, and distilled water mixed with a commercial cationic-based fabric conditioner. Distilled water dispensed from a Millipore Q-POD ${ }^{\circledR}$ was used to ensure no contaminants would negatively influence experimental results and the PDMS under test. This dispenser produces an ultrapure purity at level 1, the highest grade (Merck MillioPore Homepage 2018).

A commercial washing machine, the Bosch Exxcel Serie 4 Washing Machine WVD24520 GB, based on this work states that $58 \mathrm{~L}$ of water is used in a washer-dryer cycle and $25 \mathrm{~L}$ of water in a wash only cycle per day. For $4-5 \mathrm{~kg}$ load it states $37 \mathrm{ml}$ of detergent and $35 \mathrm{ml}$ of fabric conditioner is needed. The experimental solutions are show in Table 1.

An Eppendorf Research ${ }^{\circledR}$ plus pipette was used to dispense five $10 \mu \mathrm{l}$ droplets of distilled water, detergent, and fabric conditioner solutions upon each PDMS sample surface (Fig. 4). Each contact angle was measured via the

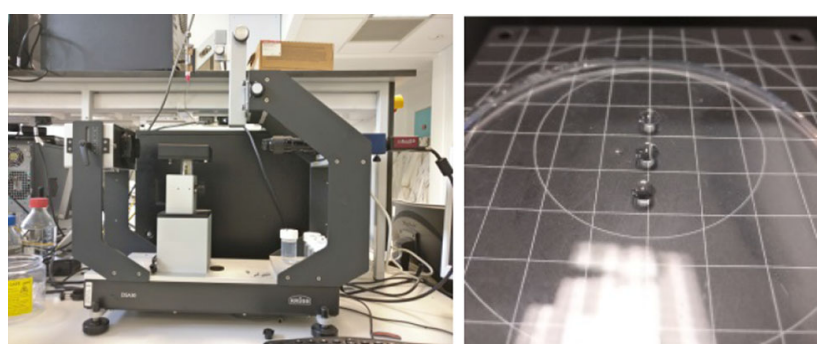

Fig. 3 Photo of the DSA30 Krüss contact angle machine $(L)$ and exemplar cured PDMS disc with droplets on surface $(R)$ 
Table 1 Aqueous ratio conversion for contact angle test

\begin{tabular}{llll}
\hline Aqueous solution & \multicolumn{2}{l}{ Aqueous conversions } & \\
\cline { 2 - 4 } & Ratio content & Commercially-recommended ratio & Experimental ratio \\
\hline DetergentA & Detergent:water & $37 \mathrm{ml}: 58 \mathrm{~L}$ & $0.32 \mathrm{ml}: 500 \mathrm{ml}$ \\
DetergentB & Detergent: water & $37 \mathrm{ml}: 25 \mathrm{~L}$ & $0.74 \mathrm{ml}: 500 \mathrm{ml}$ \\
FabricCondA & Fabric conditioner:water & $35 \mathrm{ml}: 58 \mathrm{~L}$ & $0.3 \mathrm{ml}: 500 \mathrm{ml}$ \\
FabricCondB & Fabric conditioner:water & $35 \mathrm{ml}: 25 \mathrm{~L}$ & $0.7 \mathrm{ml}: 500 \mathrm{ml}$ \\
\hline
\end{tabular}

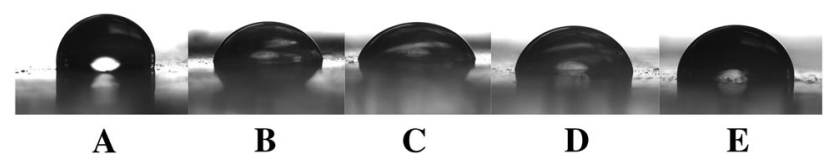

Fig. 4 Droplets upon 20:1 PDMS surface for distilled water (a), DetergentA (b), DetergentB (c), FabricCondA (d), and FabricCondB (e), captured via DropShape software

DropShape software five times to get an average, standard deviation, and error calculation.

\subsection{Swelling test aqueous permeability experiment}

This test was designed to simulate textile handwashing by submerging the PDMS strips in various washing aqueous solutions for set washing times. The detergent used was not designed for handwashing by the product manufacturer. However, it was still evaluated to establish its behavior with PDMS (Table 2).

As before, five $0.4 \mathrm{~g}$ strip-shaped PDMS samples for each mixing ratio were used. For each experimental trial, a $1.2 \mathrm{~L}$ capacity glass container was filled with either $1 \mathrm{~L}$ of distilled water, a mix of $1.8 \mathrm{ml}: 1 \mathrm{~L}$ of fabric conditioner to water (FabricCondC), or a mix of $1.8 \mathrm{ml}: 1 \mathrm{~L}$ of detergent to water (FabricCondC). Furthermore, a trial with $1 \mathrm{~L}$ tap water was investigated for further comparison. Each container had five PDMS strips which were spread out when submerged within the aqueous solutions to ensure permeation regions were not obstructed (Fig. 5).

The weight of each strip was measured before and after aqueous submerging using the KERN EMB 500-1. Any change in weight would be due to aqueous permeation into the PDMS. Aqueous submerging time of the strips for the swelling test reflected typical handwashing durations-15, 30,45 and $60 \mathrm{~min}$.

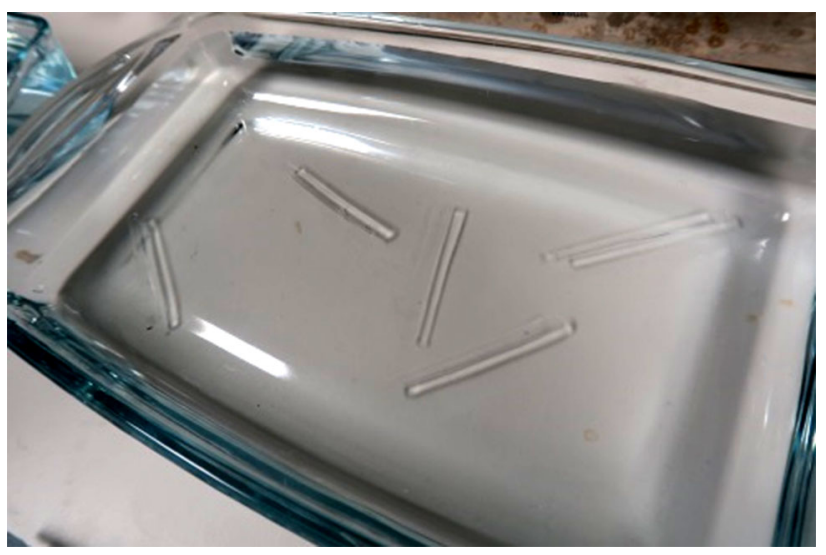

Fig. 5 Photo of swelling test setup with glass container filled with aqueous solutions with five PDMS strips of the same mixing ratio submerged

\subsection{Washing machine experiment}

Following these experiments, an average $40.3 \mu \mathrm{m}$ conformal 20:1 PDMS encapsulation was applied to four identical capacitive-based touch and proximity sensing circuits to test its functionality after multiple washing cycles. Proximity was defined as a stretched-out hand with a nominal detection distance of $2 \mathrm{~cm}$ and touch was defined as a finger pressing onto the sensing electrode portion of the circuit. Figure 6 shows an scanning electron microscope (SEM) image with measuring PDMS thickness on the circuit at two points ( $\mathrm{Pa} 1$ and $\mathrm{Pa} 2)$ to give a $40.3 \mu \mathrm{m}$ average.

Figure 7 shows a photo of one of the PDMS-packaged capacitive proximity and touch circuits that can use human interaction to illuminate an LED in a sensor-actuator configuration. The flexible circuit has a small feature size within the textile, which is helped by its 0402-packaged (metric) SMD components. It is an example circuit demonstrating the e-textile platform technology developed
Table 2 Aqueous ratio conversion for swelling test

\begin{tabular}{llll}
\hline Aqueous solution & Aqueous conversions & & \\
\cline { 2 - 4 } & Ratio content & Commercially-recommended ratio & Experimental ratio \\
\hline FabricCondC & Fabric conditioner:water & $18 \mathrm{ml}: 10 \mathrm{~L}$ & $1.8 \mathrm{ml}: 1 \mathrm{~L}$ \\
DetergentC & Detergent:water & N/A & $1.8 \mathrm{ml}: 1 \mathrm{~L}$
\end{tabular}




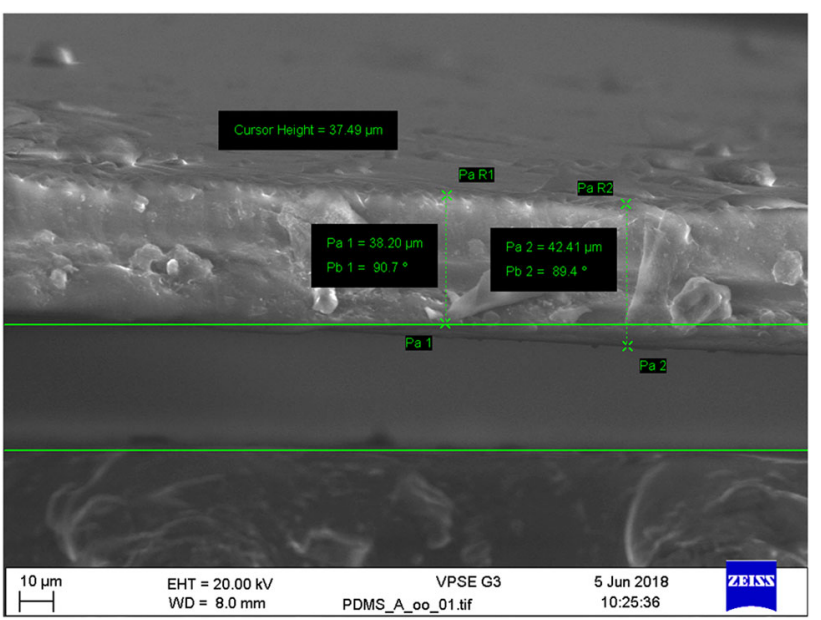

Fig. 6 SEM image of conformal PDMS layer thickness

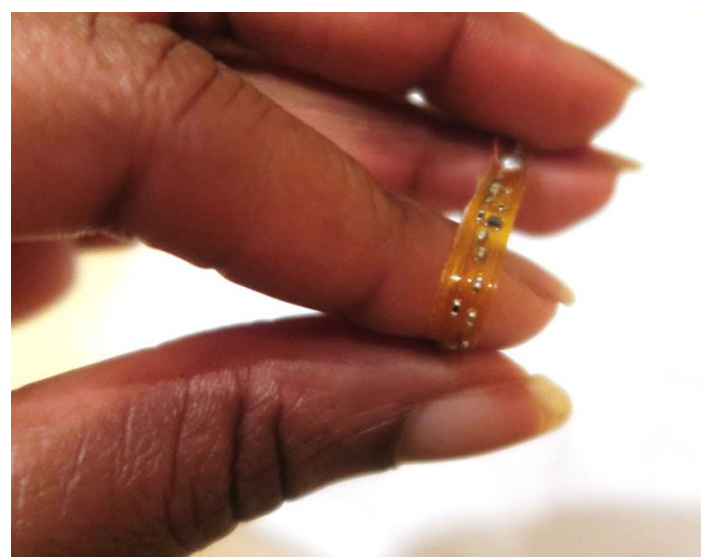

Fig. 7 Flexible conformal PDMS-packaged layer on capacitive proximity and touch sensing circuit

in the Functional Electronics TexTiles (FETT) project (FETT Project Homepage 2011) which covers the fabrication of thin, strip-shaped, flexible circuits completely integrated at yarn-level into a textile using knitting and weaving techniques.

For wearable technology and e-textile applications, capacitive sensing is stated in literature to be the most commonly-used (Frank and Kuijper 2017), most reliable (Wang et al. 2017), one of the least complex (Rus et al. 2015), low cost (George et al. 2010) and textile compatible (Jagiella et al. 2006) touch and proximity sensing method. These factors were important, because the $35 \mathrm{~mm}(\mathrm{~L}) \times 3$ $\mathrm{mm}(\mathrm{W})$ sensory circuit manufactured in this work is fully embedded into the textile-sensing through a conformal PDMS and textile layer. It relies on human body capacitance via a hand to act as the second capacitive electrode. Via self-capacitance, an electric field is created between the human hand and the sensing electrode attached to the circuit. The sensing electrode comprises of a $43 \mu \mathrm{m}$ thick flexible copper-polyimide substrate connected to the
PDMS-encapsulated, capacitive proximity and touch sensor circuit via wire. The copper-polyimide sensing plate, when provided a voltage from the circuit, becomes a selfcapacitance sensing electrode and the human hand acts as a trigger object as it causes a quanitfiable disturbance in the electric field generated. Depending on how the human hand interacts with the sensing electrode, and the circuit calibration, the circuit can sense the human hand's proximity and/or touch.

Washing machine experiments were conducted in the same consumer washing machine used to calculate the detergent and fabric conditioner ratios in the contact angle and aqueous permeability experiments. In compliance with the textile washing standard ISO 6330:2012 (Washing 2000), this was a type A washing machine i.e., a frontloading horizontal axis washing machine. The $40.3 \mu \mathrm{m}$ thick PDMS packaged sensory circuits were woven into a textile, see Fig. 8, using a hand loom and washed with $2 \mathrm{~kg}$ of cotton fabrics. This weight was compliant to ISO 6330:2012.

The e-textiles under test (Fig. 9) were subjected to three different commercial washing settings: SuperQuick $\left(15 \mathrm{~min}, 30^{\circ} \mathrm{C}\right)$, silk/delicates $\left(37 \mathrm{~min}, 30^{\circ} \mathrm{C}\right)$, and wool/ handwash $\left(42 \mathrm{~min}, 30^{\circ} \mathrm{C}\right)$. These were chosen to represent typical washing settings for textiles (Kim and Jeong 2011). $30{ }^{\circ} \mathrm{C}$ temperature is typically used for washing as most textiles are sensitive to heat. Each have different spin time changes throughout the cycle making each washing cycle different in degree of mechanical abrasion. Table 3 lists the differences between the washing settings.

Each e-textile was separated depending on the washing cycle setting they were examined against.

The same detergent and fabric conditioner used in the contact angle and aqueous permeability experiments were used. For each experimental trial, $37 \mathrm{ml}$ of detergent was poured into a cap and placed inside the washing machine with the textiles as specified by the product manufacturer.

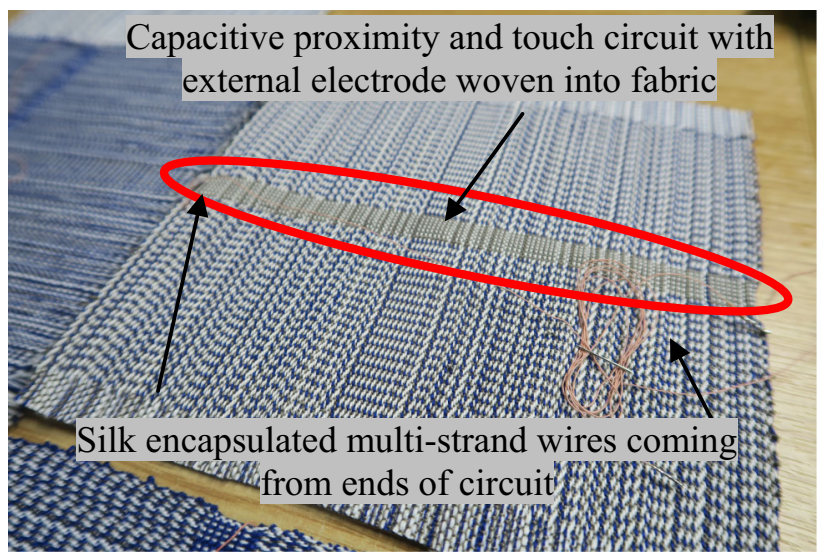

Fig. 8 Photo of e-textile samples annotated with power, ground, and integrated circuit 


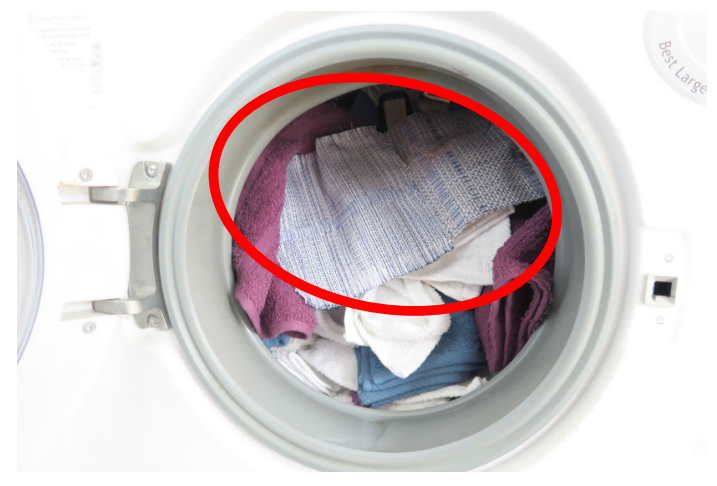

Fig. 9 Photo of e-textile samples in Bosch Exxcel Serie 4 Washing Machine WVD24520 GB

For consistency, the cotton towels and the e-textiles were placed in the washing machine in a repeatable manner: Two-thirds of the cotton towels were put in the drum initially to position the e-textiles in the middle of the drum. Then the remaining towels were placed to cover the e-textile and the detergent-filled cap was placed on top of the cotton towels. This was to ensure all the e-textile samples would be subjected to the approximately same amount of mechanical forces before the washing began.

$35 \mathrm{ml}$ of fabric conditioner was put in the middle drawer of the washing machine-this amount was appropriate for a $2-3 \mathrm{~kg}$ weight of textiles in the washing machine occurring in this experiment, following the manufacturer's guidelines.

After each wash, the circuits were dried flat on a stainless steel drying rack at a room temperature of $\sim$ $25{ }^{\circ} \mathrm{C}$ - stated as drying method C in British Standard BS EN ISO 6330:2012 in Domestic washing and drying procdures for textile washing (EN ISO 6330 2000). Then, once dried for $1 \mathrm{~h}$ and $30 \mathrm{~min}$ the circuits were tested for proximity and touch sensing functionality via an Agilent Technologies DSO3062A digital storage oscilloscope (Fig. 10).

Each e-textile circuit was powered via an EX354 Dual Power supply set to $4.5 \mathrm{~V}$ and maximum 0.03 A current. An X10 oscilloscope probe was connected between the circuit's ground and the copper electrode via a test wire soldered on to it before it was integrated into a textile. Using this method, it is possible to acquire the RC

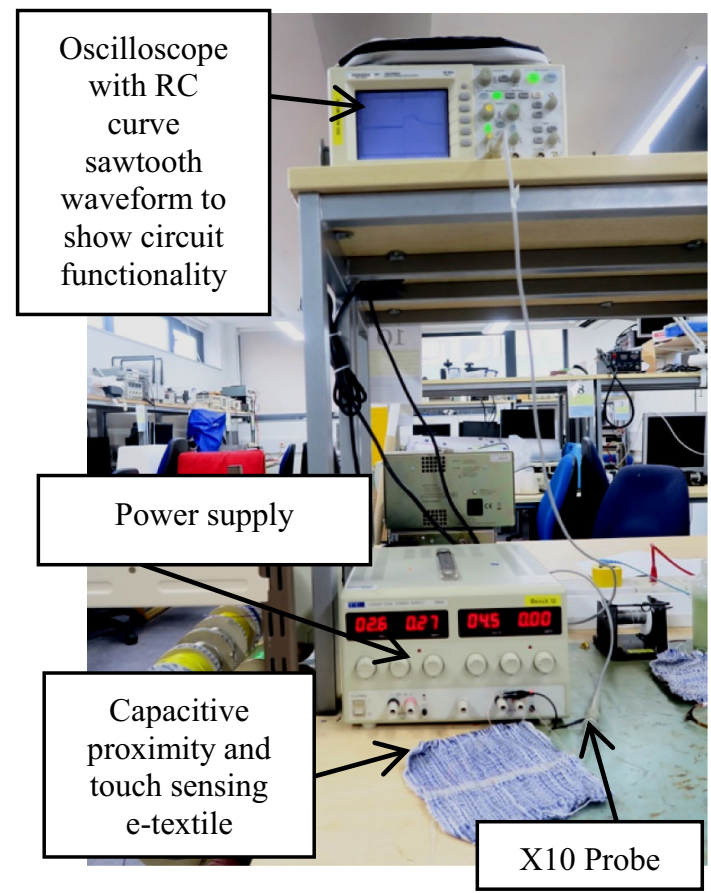

Fig. 10 Testing the e-textile with a power supply and oscilloscope to monitor the output

characteristics of the copper self-capacitance sensing electrode (Figs. 11 and 12). This RC characteristic is produced by the approximately $4.5 \mathrm{~V}$ source provided through the proximity chip charging the self-capacitive copper electrode via a $6.8 \mathrm{k} \Omega$ resistor also at the chip input.

The touch proximity chip used in the e-textile circuit (Fig. 13) is the PCF8883US by NXP Semiconductor. It has the external sensing electrode at its input connected to an internal $\mathrm{RC}$ timing circuit. The discharge time of the internal RC timing circuit is compared to a synchronised, second internal RC timing circuit that is used as a reference. Proximity and/or touch are registered by the chip when the discharge time is longer than the reference RC timing circuit. Hence, the time delay for the proximity chip when it responds to a proximity or touch equivalent signal is known as the time constant. It takes approximately five time constants during the transient response to fully charge or discharge a capacitor before reaching a steady.

Table 3 Washing settings from BOSCH Exxcel Serie 4 washing machine WVD24520 GB

\begin{tabular}{|c|c|}
\hline $\begin{array}{l}\text { Washing } \\
\text { setting }\end{array}$ & Explanation \\
\hline SuperQuick & Short, 15 min cycle typically set at the highest washing speed of $1200 \mathrm{rpm}$. Typically used on cotton textiles \\
\hline Silk/delicates & Fabrics spun at the lowest speed of the washing machine ( $400 \mathrm{rpm})$, used to wash silk, viscose, and satin textiles \\
\hline $\begin{array}{l}\text { Wool/ } \\
\text { handwash }\end{array}$ & $\begin{array}{l}\text { Fabrics spun at lowest speed at washing machine }(400 \mathrm{rpm}) \text {, to prevent woolens from matting due to moisture and heat the } \\
\text { washing machine alternates from spinning and standing still }\end{array}$ \\
\hline
\end{tabular}




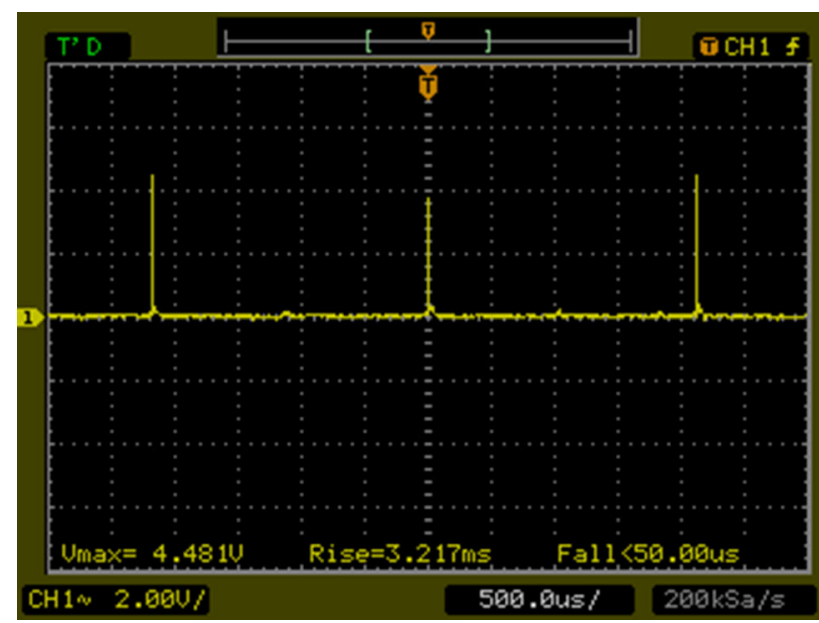

Fig. 11 Initial oscilloscope waveform generated when circuit is given power, showing three periodic outputs

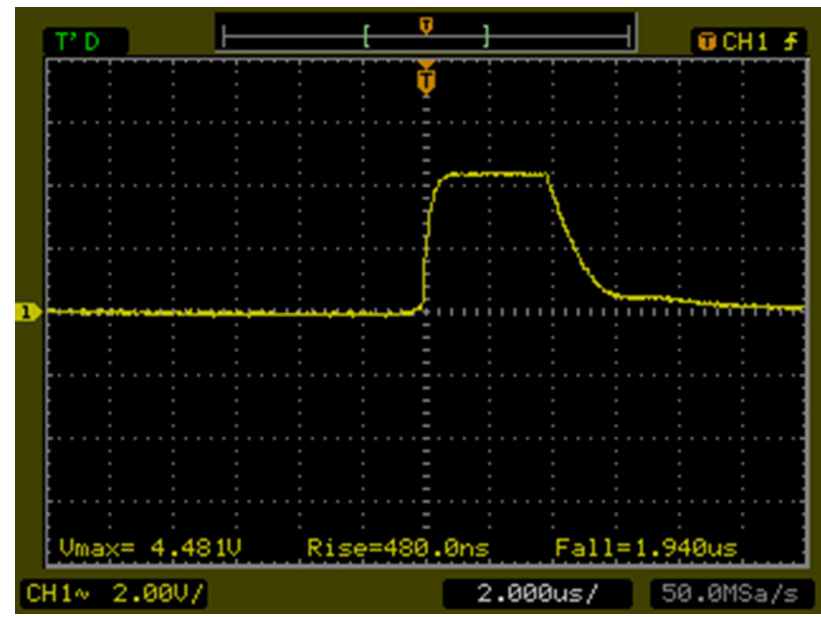

Fig. 12 Zooming into one of the outputs in the initial oscilloscope waveform shows the RC curve response showing the maximum capacitive charging voltage $\left(V_{\max }\right)$, the capacitor charging time (rise time), and capacitor discharging time (fall time) of capacitive sensory e-textile

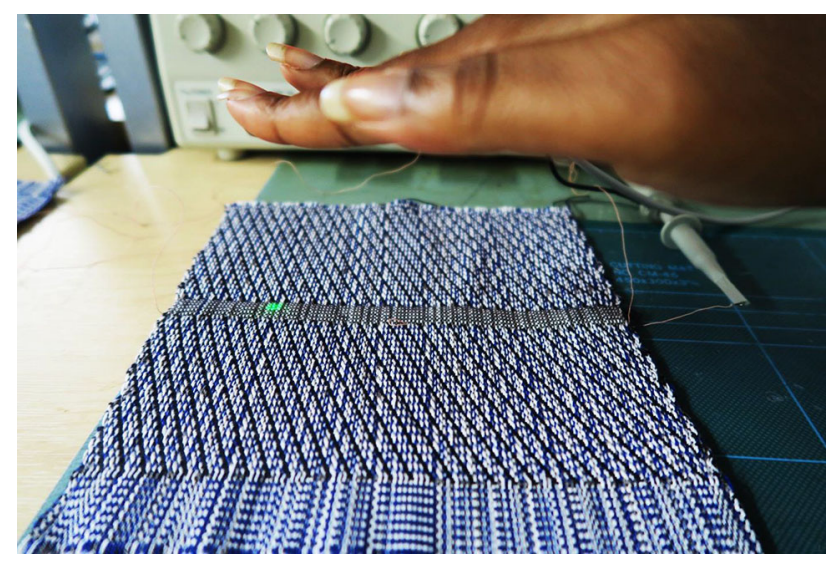

Fig. 13 Photo of e-textile circuit green LED illuminating before being washed as visual feedback of functionality of proximity sensing

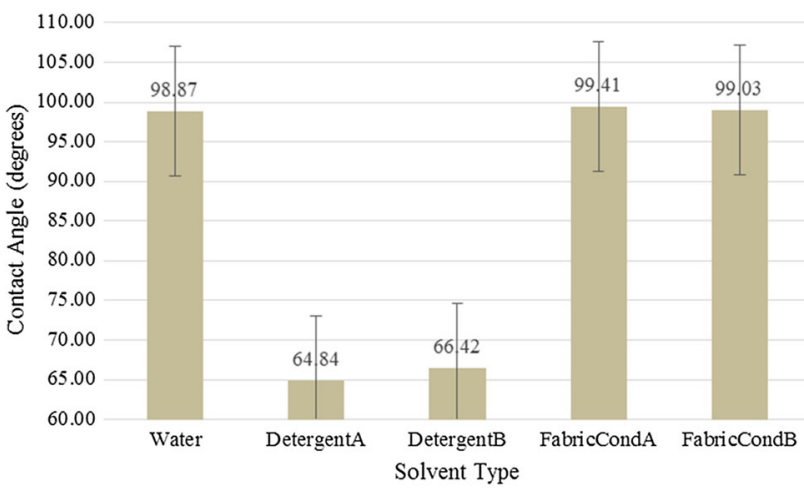

Fig. 14 5:1 PDMS contact angle measurements to water, detergent, and fabric conditioner solutions

This means that as the capacitor connected to the sensor input, which is connected to the copper sensing electrode integrated into the textile, increases then the discharge time also increases. This will cause an increase in rise and fall time-full charge and discharge time of the capacitor respectively. Additionally, as the electric field strength between the copper electrode and the human hand increases as the hand moves closer to the electrode it is expected that the rise time and fall time will increase. As a measure of the e-textile's sensing efficiency it is also anticipated that the amount of charging voltage will decrease as the number of washing cycles increases as the self-capacitance copper sensing plate becomes less conductive. These three factors — rise time, fall time, and charging voltage —of the self-capacitance copper electrode is obtained by the oscilloscope by measuring the $\mathrm{RC}$ curve and using this to determine the sensing efficiency.

Circuit functionality checks using the oscilloscope were performed after the first wash to check survival, then with every fifth washing interval i.e., 5, 10, 15, 20. This methodology was used in previous literature (Merritt et al. 2009; Varnaite and Katunskis 2009; Kazani et al. 2012; Kaappa et al. 2017; Satharasinghe et al. 2018) to test electronic textile functionality after multiple washing cycles to evaluate durability and robustness. Values for rise time, fall time, and maximum charging voltage seen at pin 1 were recorded five times for each e-textile under test and an average was calculated (Fig. 14).

\section{Experimental results}

\subsection{Contact angle hydrophobicity experiment}

Results showed decreasing the cross-linking of PDMS has an effect on how the water, detergent, and fabric conditioner droplets behave upon its surface (Figs. 15, 16, 17, 18 and 19). Overall, the contact angle increases when cross- 


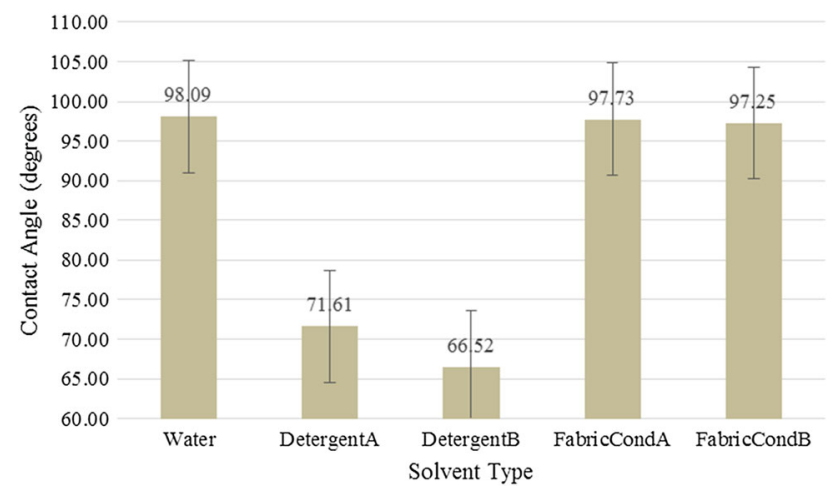

Fig. 15 7:1 PDMS contact angle measurements to water, detergent, and fabric conditioner solutions

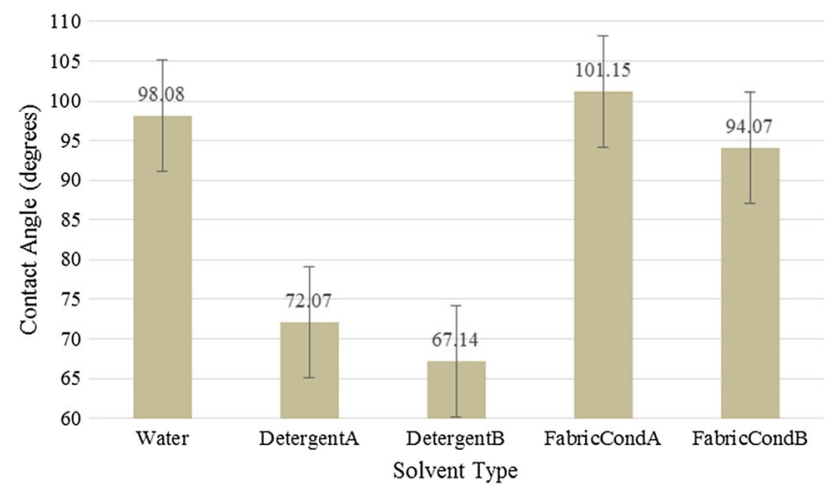

Fig. 16 10:1 PDMS contact angle measurements to water, detergent, and fabric conditioner solutions

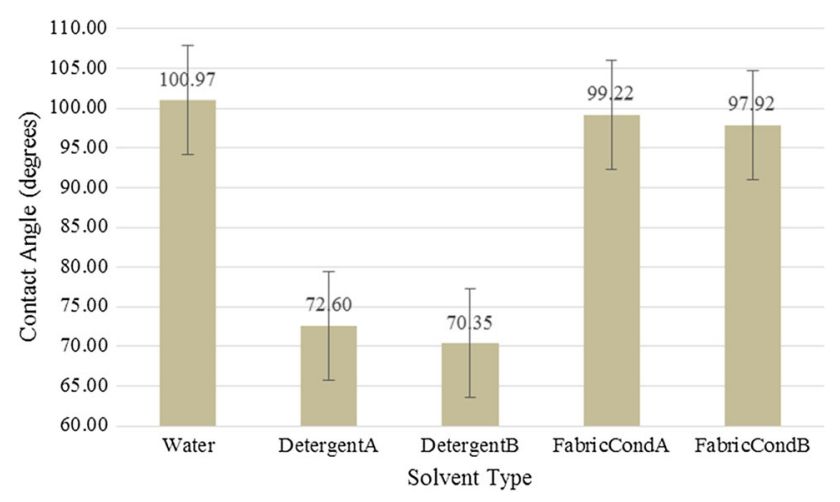

Fig. 17 15:1 PDMS contact angle measurements to water, detergent, and fabric conditioner solutions

linking reduces despite the concentration of surfactants increasing.

Despite the changing surface tension of the solutions due to the concentration of surfactants, a PDMS mixing ratio of 20:1 overall gives the greatest contact angle values compared to other mixing ratios. This is expected, as reduced the cross-linking increases the number of excess silicon-hydride groups which prevents charged hydroxide ions to the surface.

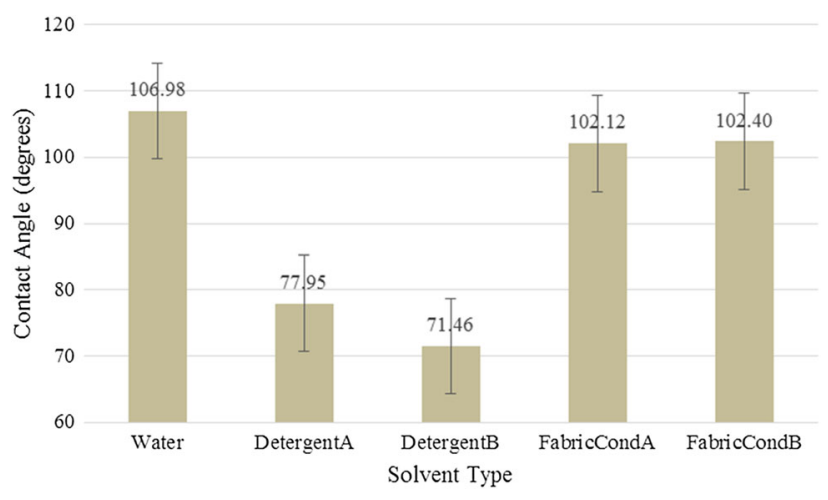

Fig. 18 20:1 PDMS contact angle measurements to water, detergent, and fabric conditioner solutions

Additionally, detergent has a greater concentration of surfactants compared to fabric conditioner which encourages aqueous solutions to adsorb onto interfacing surfaces (Essö 2007). This explains why the contact angles for detergent are lower as surfactants reduce the surface tension of the aqueous solution as it interfaces with surfaces during the washing process. Expectedly, detergent and fabric conditioner display lower contact angles compared to water. This shows that PDMS is compatible to these chemicals as it does not disrupt the desired functionality of detergent and fabric conditioner upon textiles-still allowing the adsorbing onto textiles to clean it—whilst still protecting the electronics that would be encapsulated within it.

For all aqueous solutions tested, 20:1 was the most hydrophobic with an $8.3 \%, 7.5 \%, 6 \%, 1 \%$, and $8.1 \%$ improvement compared to 10:1 for distilled water, DetergentA, DetergentB, FabricCondA, and FabricCondB respectively. Although measurements were similar-indicating similar surface energy between mixtures-they suggest that decreasing the crosslinking of the PDMS structure increases hydrophobicity to water, which is supported by literature (Palchesko et al. 2012). However, this paper expands the literature by showing this trend is the same when using detergent and fabric conditioner solutions, not just water. Furthermore, measured contact angle values were within $10 \%$ of those reported in literature (Palchesko et al. 2012) increasing confidence in these findings.

\subsection{Swelling and aqueous permeability experiment}

Change in weight of the PDMS strips when submerged in distilled water, tap water, FabricCondC, and DetergentC is evident by calculating the degree of swelling (Lee et al. 2003) as in equation (1) from (Honda et al. 2005), 


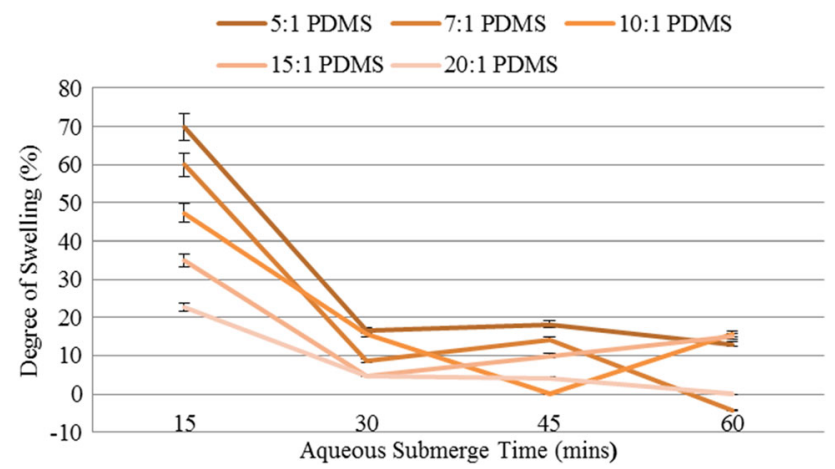

Fig. 19 Average degree of swelling (\%) reduces with time overall for five PDMS mixing ratios submerged in distilled water

Degree of swelling (\%)

$$
=\frac{\text { Increased weight }(\mathrm{g})-\text { Initial weight }(\mathrm{g}) \times 100}{\text { Initial weight }(\mathrm{g})} .
$$

Swelling tests conducted in distilled water showed a significant improvement in reducing aqueous permeation of PDMS as the cross-linking decreased. Similar results show that mixing ratio has an influential factor regarding swelling in FabricCondC and DetergentC.

By using 20:1 instead of 10:1, experiment recorded that the degree of swelling in water can reduce by $48 \%$ (Table 4). Additionally, as the duration of swelling increases, the degrees of swelling decreases until negligible or zero.

Degree of swelling in FabricCondC, are comparably less compared to the distilled water experiment (Table 5), whereby the behaviour crosslinking affecting aqueous permeability is overall inconclusive (Fig. 20). Instead, it appears swelling increases for every $15 \mathrm{~min}$ cycle for all mixing ratios except 20:1 which alternates every $30 \mathrm{~min}$. This irregularity is possibly due to the surfactants encouraging more surface than permeability reaction.

Similarly, for DetergentC the degree of swelling reduces as the aqueous submerge time increases overall (Fig. 21) for 20:1 and 15:1. Although at duration times 20:1 has the greatest degree of swelling at $15 \mathrm{~min}$ as time increases it is

Table 4 Average contact angle measurements

\begin{tabular}{lllrrr}
\hline Aqueous solution & \multicolumn{6}{l}{ Contact angle (degrees) } \\
\cline { 2 - 6 } & \multicolumn{1}{l}{$5: 1$} & \multicolumn{1}{l}{$7: 1$} & \multicolumn{1}{c}{$10: 1$} & \multicolumn{1}{c}{$15: 1$} & \multicolumn{1}{c}{$20: 1$} \\
\hline Water & 98.87 & 98.09 & 98.08 & 100.97 & 106.98 \\
DetergentA & 64.84 & 71.61 & 72.07 & 72.60 & 77.95 \\
DetergentB & 66.42 & 66.52 & 67.14 & 70.35 & 71.46 \\
FabricCondA & 99.41 & 97.73 & 101.15 & 99.22 & 102.12 \\
FabricCondB & 99.03 & 97.25 & 94.07 & 97.92 & 102.40 \\
\hline
\end{tabular}

Table 5 Average swelling test results for distilled water

\begin{tabular}{llccc}
\hline PDMS mixing ratio & \multicolumn{4}{l}{ Degree of swelling (\%) 1 d.p. } \\
\cline { 2 - 5 } & 15 min & 30 min & 45 min & 60 min \\
\hline $5: 1$ & 70.0 & 16.7 & 18.2 & 13.0 \\
$7: 1$ & 60.0 & 8.7 & 14.3 & 4.2 \\
$10: 1$ & 47.4 & 15.8 & 0.0 & 15.8 \\
$15: 1$ & 35.0 & 4.8 & 10.0 & 15.0 \\
$20: 1$ & 22.7 & 4.8 & 4.2 & 0.0 \\
\hline
\end{tabular}

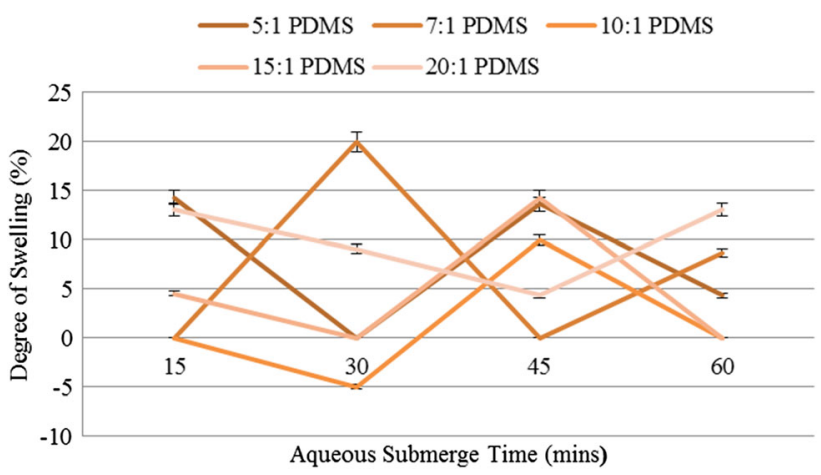

Fig. 20 Average degree of swelling (\%) reduces with time with 20:1, and reduces every $15 \mathrm{~min}$ alternate with 5:1, 7:1 and 15:1 PDMS strips in FabricCondC solution

least affected by aqueous solution compared to other crosslinked PDMS types (Table 6). Interestingly, a similar behavior occurs whereby swelling increases ever $30 \mathrm{~min}$ cycle for 5:1, 7:1, and 20:1.

A further trial evaluating PDMS' reaction with tap water was also conducted-making results more representative of handwashing. Similarly, to DetergentC and FabricCondC, tap water also shows that with increased aqueous submerge time the degree of swelling reduces for 30 min intervals (Table 7).

Compared to 10:1, 20:1 PDMS has a $45.5 \%$ reduction in swelling-similar to distilled water. As the time duration

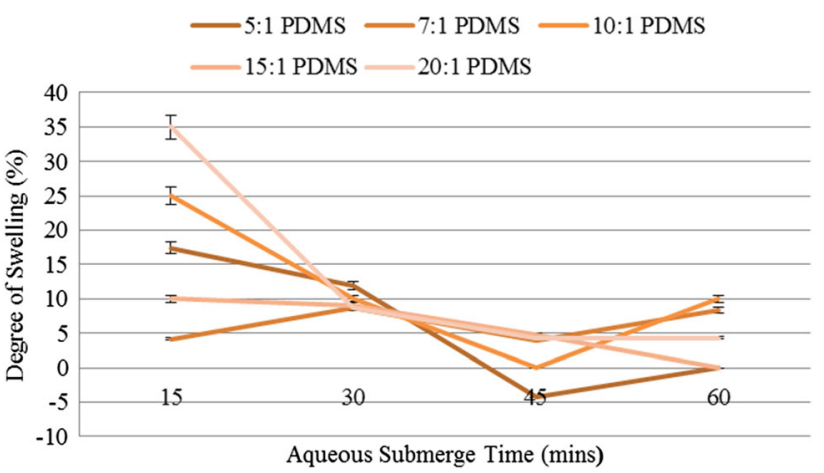

Fig. 21 Average degree of swelling (\%) reduces with time all PDMS fabrications under test in DetergentC solution 
Table 6 Average swelling test results for FabricCondC

\begin{tabular}{lrrrr}
\hline PDMS mixing ratio & \multicolumn{4}{c}{ Degree of swelling (\%) 1 d.p. } \\
\cline { 2 - 5 } & $15 \mathrm{~min}$ & $30 \mathrm{~min}$ & $45 \mathrm{~min}$ & $60 \mathrm{~min}$ \\
\hline $5: 1$ & 14.3 & 0.0 & 13.6 & 4.3 \\
$7: 1$ & 0.0 & 20.0 & 0.0 & 8.67 \\
$10: 1$ & 0.0 & -5.0 & 10.0 & 0.0 \\
$15: 1$ & 4.6 & 0.0 & 14.3 & 0.0 \\
$20: 1$ & 13.0 & 9.1 & 4.3 & 13.0 \\
\hline
\end{tabular}

Table 7 Average swelling test results for DetergentC

\begin{tabular}{lcccc}
\hline PDMS mixing ratio & \multicolumn{5}{c}{ Degree of swelling $(\%)$} \\
\cline { 2 - 5 } & $15 \mathrm{~min}$ & $30 \mathrm{~min}$ & $45 \mathrm{~min}$ & $60 \mathrm{~min}$ \\
\hline $5: 1$ & 17.4 & 12.0 & 4.2 & 8.3 \\
$7: 1$ & 4.2 & 8.7 & 4.0 & 10.0 \\
$10: 1$ & 25.0 & 10.0 & 0.0 & 0.0 \\
$15: 1$ & 10.0 & 9.1 & 4.8 & 4.3 \\
$20: 1$ & 35.0 & 8.7 & 4.3 & 8.3 \\
\hline
\end{tabular}

increases, the degree of swelling reduction of 20:1 compared to $10: 1$ becomes $10 \%, 28.7 \%$ and $25 \%$.

The result that degree of swelling reduces with aqueous submerge time at all may seem counter-intuitive as it is expected to increase for typical porous materials. However, PDMS is hydrophobic which has a replant effect on its interaction with aqueous solution at pore-level. PDMS can be synthesised to have specific mechanical or chemical characteristics enhanced for its application (Brook and Organic 2000) by changing its cross-linking, fabrication method, or thickness (Schirhagl and Zare 2011) can control the porosity attributes of PDMS. Contact Angle tests suggested that reduced cross-linking increased PDMS' hydrophobicity. Consequently, experiments indicate that PDMS' degree of swelling reduces overall as cross-linking reduces when in water, hence as PDMS becomes more hydrophobic. However, in detergent and fabric conditioner this degree of swelling alternates at set time intervals. As a result, this appears as PDMS having alternating degree of swelling and the set alternating time period depends on the aqueous solution the PDMS is submerged in Table 8, with corresponding graph Fig. 22.

The reduced degree of swelling of PDMS in fabric conditioner and detergent solutions compared to water may be due surface action. Detergent (anionic surfactants) and fabric conditioner (cationic or anionic surfactants) contains cleaning agents which reduce the surface tension of water, enabling water to penetrate the surface of oil, and stain particles into finer sizes as part of their removal process. Anionic surfactants have a negatively charged hydrophilic
Table 8 Average swelling test results for tap water

\begin{tabular}{lllll}
\hline PDMS mixing ratio & \multicolumn{4}{l}{ Degree of swelling $(\%)$} \\
\cline { 2 - 5 } & $15 \mathrm{~min}$ & $30 \mathrm{~min}$ & $45 \mathrm{~min}$ & $60 \mathrm{~min}$ \\
\hline $5: 1$ & 26.1 & 21.7 & 17.4 & 17.4 \\
$7: 1$ & 29.2 & 8.3 & 22.7 & 26.1 \\
$10: 1$ & 20.0 & 10 & 15.0 & 25.0 \\
$15: 1$ & 9.1 & 9.1 & 0.0 & 25.0 \\
$20: 1$ & 9.1 & 0.0 & 4.3 & 0.0 \\
\hline
\end{tabular}

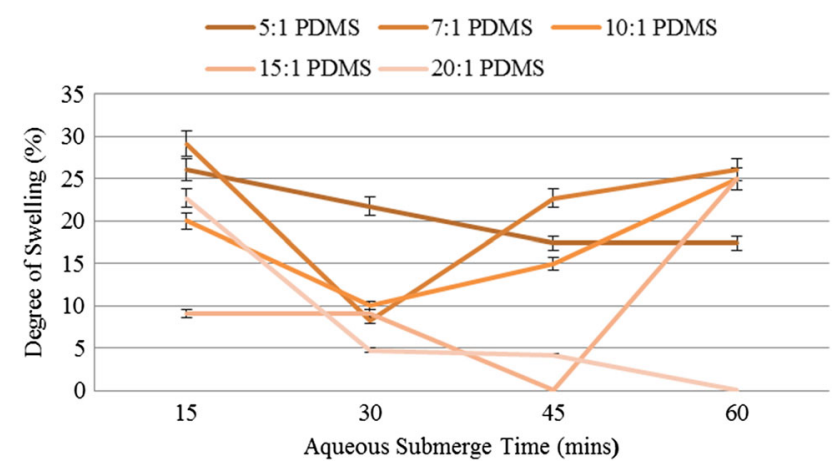

Fig. 22 Average degree of swelling (\%) reduces every $30 \mathrm{~min}$ for PDMS fabrications under test in tap water

end whereas cationic surfactants have a positively charged hydrophilic end. For anionic surfactants, the negativelycharged hydrophilic ends position themselves away from the dirt, oil, and/or stain molecules to help suspend them to the liquid surface, whilst the hydrophobic end is attracted to the dirt and oil molecules and helps forms the micelle. Cationic surfactants have a positively-charged hydrophilic end. The hydrophobic surface of PDMS could prevent the degree of hydrophobic portions of the surfactant molecules that could reach the surface. As a result, fabric conditioner and detergent encourages more surface action that relatively reduces permeation of aqueous solution which would theoretically explain the experimental results. Accordingly, when PDMS is submerged in an aqueous solution with a weaker surface tension, this reduces degree of aqueous solution that would otherwise be on its surface.

Additionally, some fabric conditioners feature sodium silicates which produce a lubricating, protective layer over the tank and drum of the washing machine. This protective lubricating layer is also reported in literature (Brooks et al. 1989) to be present on the items in the wash. This is a type of chemical finish of surfactants, whereby fabric conditioners especially contain long chain fatty acids that soften fabrics to make them appear more smooth and soft to touch. It is possible that the fatty acid layer (Ren et al. 2017) lubricates the PDMS whilst submerged in aqueous solution creating a buffer preventing aqueous permeation. Therefore, the phenomena described may have caused the 
minimised weight gain of the cured PDMS strips in FabricCondC and DetergentC-related to negligible degree of swelling. Nonetheless, fabric conditioner and detergent only feature in machine washing towards the end of a washing cycle to be removed and replaced with water in the final spinning process (Kang and Kim 2001). Therefore, results for water should take more importance which means experiments suggest that $20: 1$ is the better mixing ratio choice rather than 10:1 to become a hydrophobic packaging for washable microsystems integrated into textiles. Importantly, all PDMS samples reverted back to their original mass 1-3 s after removal from the solution-due to the aqueous solvents evaporating from the polymer. This suggests the PDMS will retain its initial flexibility and robustness after drying. Overall, results show PDMS' compatibility with these aqueous solutions allowing use in machine washing thus confirming its suitability as a flexible, hydrophobic electronic packaging choice for flexible electronic microsystems integrated into textiles.

\subsection{Washing machine experiment}

Comparing the difference between the e-textiles before they were subjected to washing tests (Fig. 23) and after (Fig. 24), we can visibly deduce that the integrated circuity has experienced bending and twisting. The e-textiles would have swelled during the wash due its yarns absorbing water and other aqueous solutions in the washing cycle. However, once dried, they did not return to a completely flat state and soft state. This is likely due washing the e-textile multiple times in a very short time period, causing the textile to contort.

Before subjected to washing in the washing machine, the functionality of all the e-textile samples under investigation were verified by oscilloscope with the generation of the RC curve and measurements of its rise time, fall time, and charging voltage $\left(\mathrm{V}_{\mathrm{c}}\right)$.

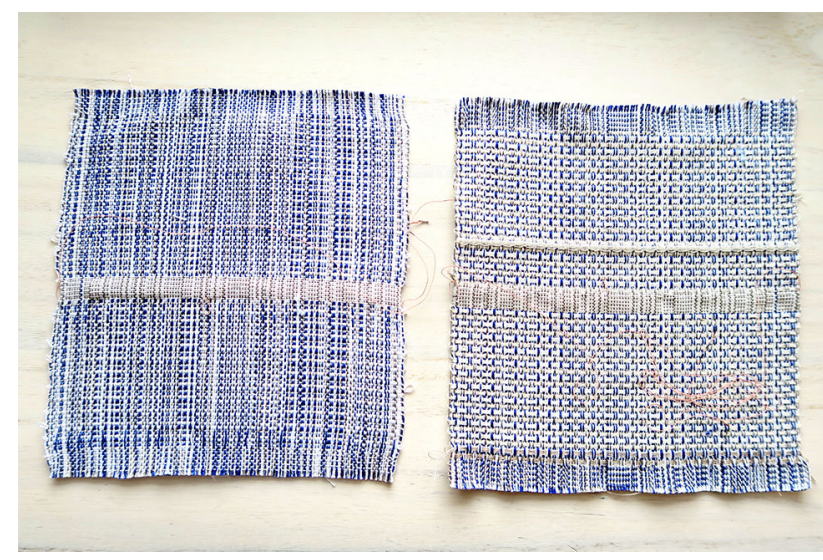

Fig. 23 Photo of some e-textile samples before washing

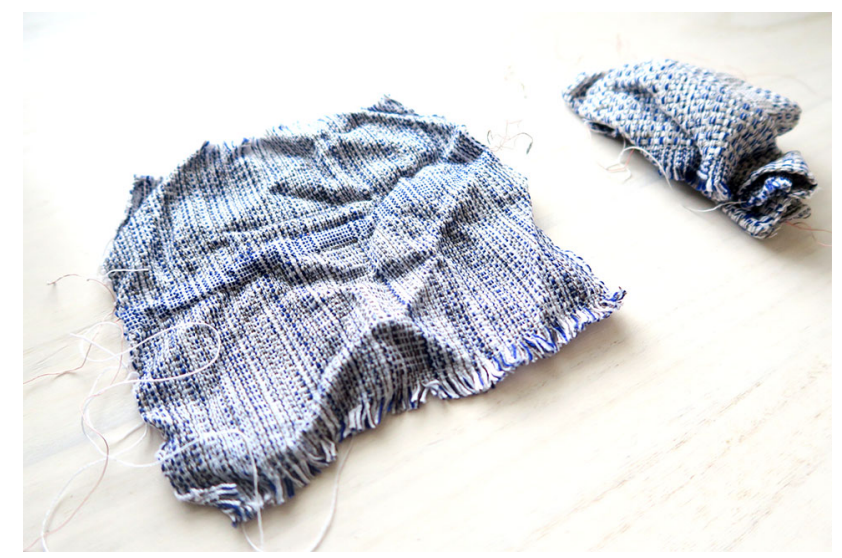

Fig. 24 Photo of e-textile samples after washing showing the sample stretched out (left) and sample deformed shape after washing cycle (right)

Before washing, it is anticipated that the maximum charging voltage $\left(\mathrm{V}_{\mathrm{c}}\right)$ of the RC curve will not match the supply voltage $\left(\mathrm{V}_{\mathrm{ss}}\right)$ value-which would occur in an ideal case. This is because some energy is stored in the capacitor at input pin of the circuit. This is due to lost energy from the capacitor used to function. This explains why the initial maximum charging voltage of all the e-textiles is less than the supply voltage of $4.5 \mathrm{~V}$.

However, Figs. 25, 26, and 27 shows overall the maximum charging voltage decreasing as the number of washes increases. This occurs for non-detection and successful proximity and touch detection of a human hand. The likely reason for this is damage to the circuit as otherwise it is expected that the voltage difference to vary negligibly if not interfacing with water or not mechanically deformed. The potential cause of this decline could be damage to the encapsulation. For the first two circuits under test, which were washed together for $15 \mathrm{~min}$ at $800 \mathrm{rpm}$ at $30{ }^{\circ} \mathrm{C}$, both circuits no longer functioned after the first wash. The fact that both circuits failed at the same time whilst being washed under the same conditions indicates that other e-textile samples would have failed. The washing speed of $800 \mathrm{rpm}$ was perhaps too high and was presumed to impose detrimental mechanical abrasions and forces.

Due to results from the contact angle and aqueous permeability tests, the failure was likely not due to the hydrophobicity of the packaging but the mechanical strain of the washing cycle process. Therefore, subsequent tests conducted washing tests at the lowest spin speed of the washing machine $-400 \mathrm{rpm}$. For washing tests completed at $400 \mathrm{rpm}$ at $37 \mathrm{~min}$ and $42 \mathrm{~min}$ at $30^{\circ} \mathrm{C}$ these circuits survived between 10 and 15 washes. The touch and proximity sensing efficiencies of all the e-textiles under test were compared:

The error bars on these graphs show the percentage error for each data point, which is an average of five readings. 


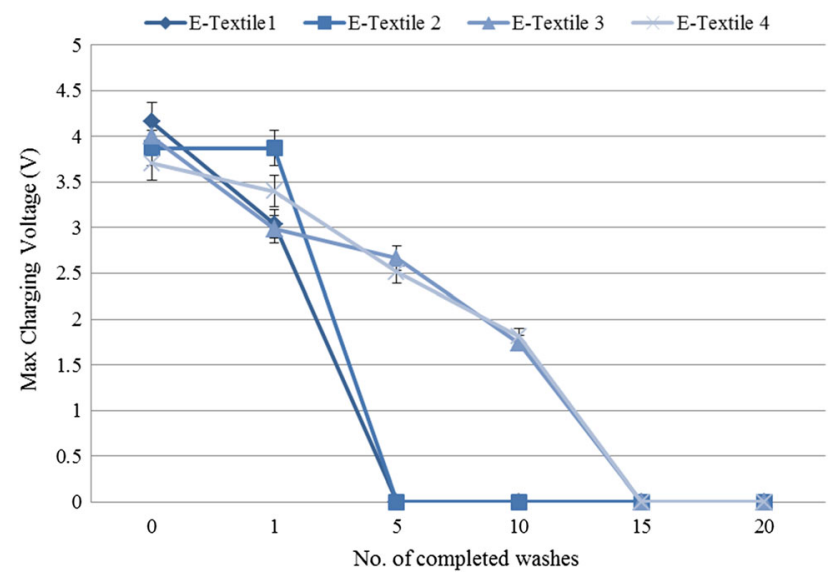

Fig. 25 Graph comparing maximum charging voltages of e-textile circuits after being washed in successive cycles when there is no detection of a trigger object (human hand)

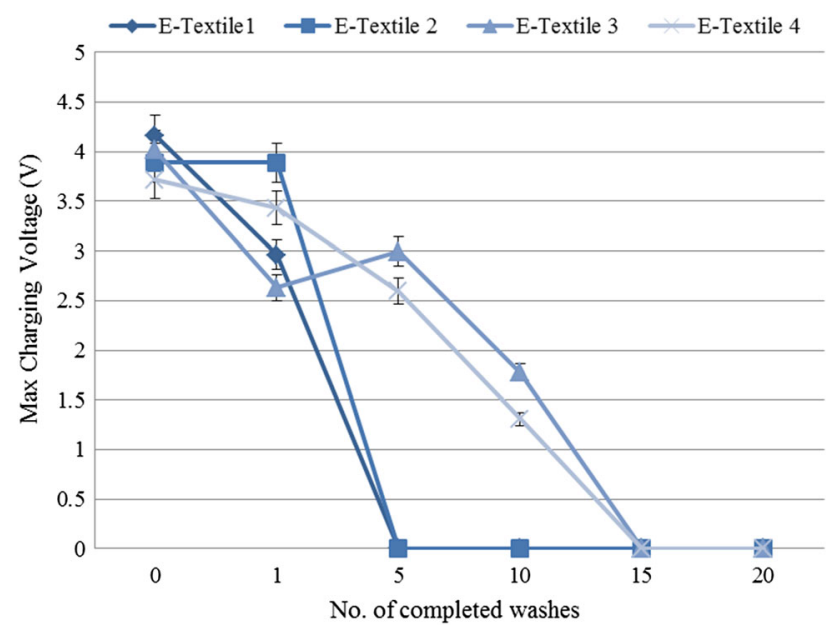

Fig. 26 Graph comparing maximum charging voltages of e-textile circuits after being washed in successive cycles when there is proximity detection of a trigger object (human hand)

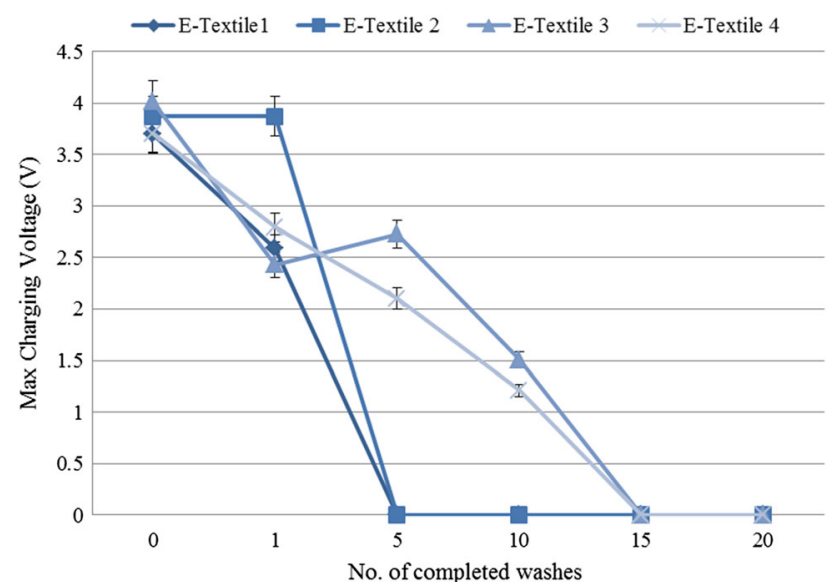

Fig. 27 Graph comparing maximum charging voltages of e-textile circuits after being washed in successive cycles when there is touch detection of a trigger object (human hand)
Examining the maximum charging voltage for proximity and touch detection (Figs. 26 and 27) the graphs also reveal that some washing settings are more detrimental than others. With the longest surviving textiles-e-textile 3 and 4-their rate of change of difference suggests one washing setting is more detrimental than the other. Both e-textiles were washed at a lower washing spin speed at $400 \mathrm{rpm}$ but at different washing settings. The washing machine manufacturer states the difference between silk/delicates (etextile 3) and wool/handwash (e-textile 4) settings is alternate pause and spin motion, thus a difference in mechanical stress experienced by the e-textile under test. As the number of washing cycles increases, the difference between the e-textile 3 and e-textile 4 increases until the circuits stop functioning which is represented on the graphs as $0 \mathrm{~V}$. Yet, as the rate of change for maximum charging voltage between the 1st and 5th wash is greater for e-textile 1 and 2 compared to 3 and 4 this suggests that the washing settings for e-textiles 1 and 2 contribute to a faster decline of circuit performance and functionality over time.

As expected, as the number of washing cycles increase, the rise and fall time of the generated RC curve increases. However, Figs. 28, 29, 30 also show that as the e-textiles are washed for longer, the sensing functionality of the circuit becomes increasingly limited. In fact, one sensing functionality increasing declines with each wash-proximity. This is supported by the RC curve rise and fall time values in the washing test. The results show that 'touch' has a greater rise and fall time value compared to 'proximity' and 'no trigger object' values which appear negligible on the graphs.

By isolating results for e-textile 3 and 4 which survived the greatest number of cycles and operated at the lowest washing cycle rotary spin speed of $400 \mathrm{rpm}$, it is observable that the circuit behaves better as a touch sensor rather than a proximity sensor due to the washing process.

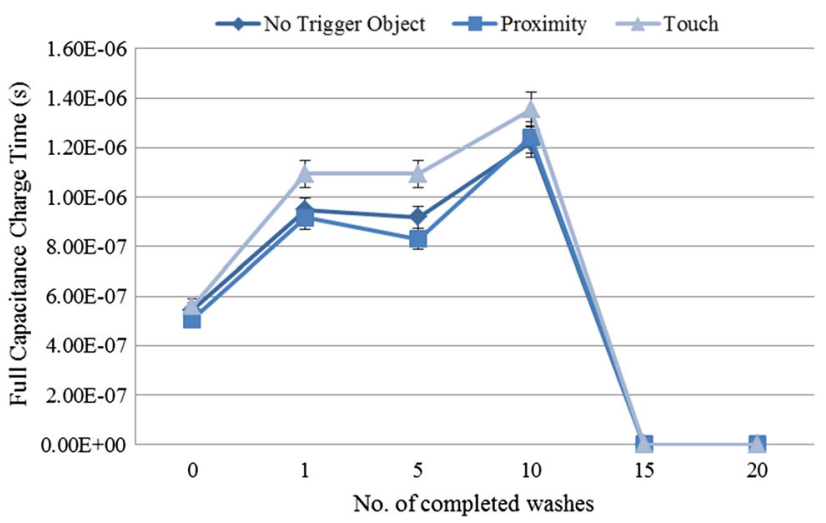

Fig. 28 Graph comparing rise time (full charging time) of e-textile 3 capacitive circuit when there is no detection, proximity detection, and touch detection of a human hand after multiple washing cycles 


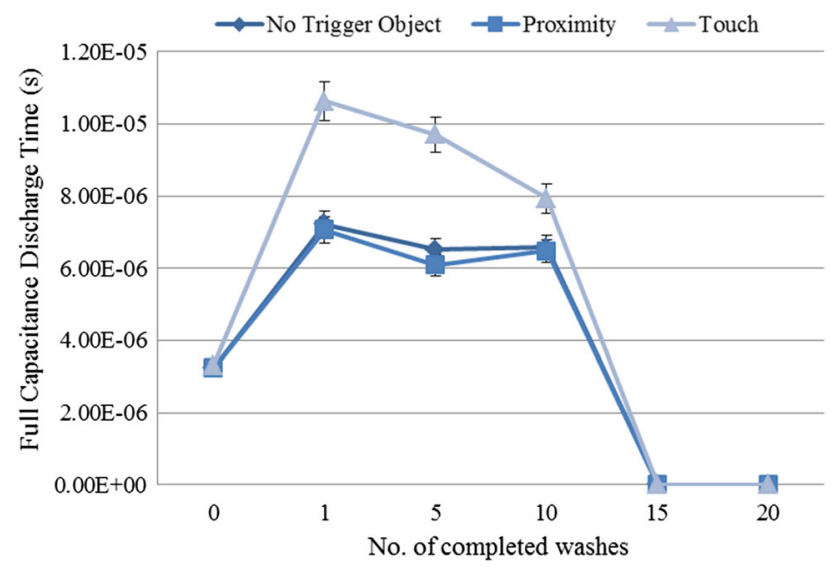

Fig. 29 Graph comparing fall time (full discharging time) of e-textile 3 capacitive circuit when there is no detection, proximity detection, and touch detection of a human hand after multiple washing cycles

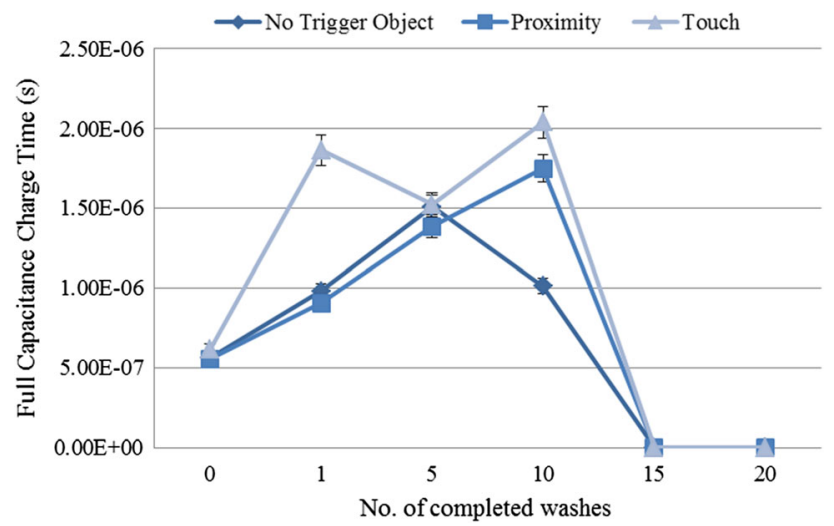

Fig. 30 Graph comparing rise time (full charging time) of e-textile 4 capacitive circuit when there is no detection, proximity detection, and touch detection of a human hand after multiple washing cycles

Looking at the percentage change in rise time values, with e-textile 3's first wash the rise time proximity detection value increases by $81 \%$ and on the tenth wash $145 \%$ compared with before washing. Compare this to touch, whereby for the first wash the change is $95 \%$ and on the tenth wash becomes $142 \%$ compared with before washing. By looking at Fig. 30 the same behavior shows that the copper sensor plate takes longer to discharge with more washing cycles which is an indication of the speed at which it becomes able to sense a trigger object i.e., human hand.

Comparably, for fall time values there is a $118 \%$ change in fall time for proximity detection comparing the before washing value with the first wash and a $100 \%$ change compared to the tenth wash. Compared to touch detection, whereby there is a $220 \%$ change in fall time for proximity detection comparing the before washing value with the first wash and a $139 \%$ change compared to the tenth wash. Regardless of these significant increases in rise time and times, the circuit is still functioning as expected. This is because capacitive theory suggests percentage change to be greater for touch as the electric field concentration is stronger. This is due to the zero distance between plates of the capacitor- the integrated copper electrode and human hand. The increase in rise time value means that the time taken for the internal capacitor connected to the copper sensor plate at the IC's input pin takes longer to charge.

Therefore, the results could deduce that independent of the washing cycle duration but likely dependent on the washing machine spin speed and temperature- the process of washing reduces the ability of the circuit to sense a trigger object. Consequently, it becomes harder for the circuit to detect the proximity of a trigger object compared to touch.

The consistency of this result is also shown with e-textile 4-whereby the rise and fall times of the circuits under test steadily increase with increased washing cycles. The circuit performs touch detection more efficiently than proximity sensing with increasing number of washes, as revealed by the percentage differences. Referring to Fig. 30, e-textile 4's first wash percentage change value for capacitive rise/ charging time proximity detection increases from the 'before washing' value by $64 \%$ and from the tenth wash value by $216 \%$. Compare this to touch, whereby for the first wash's percentage change difference value compared to 'before washing' is $202 \%$ and for the tenth wash this value becomes $230 \%$. These values are much greater than that for proximity sensing. Apart from the data point on the fifth wash in Fig. 30, touch produces a clear difference and hence longer discharge time compared to proximity and no trigger object trend lines. The data point on the fifth wash on Fig. 30 appears to be an anomaly, but appears again on the fourth e-textile shown in Fig. 31.

The same restorative behavior is noticeable on the fall time graph for e-textile 4 . This could be due to a variable oxidized layer on the sensing electrode integrated into the textile during the drying process.

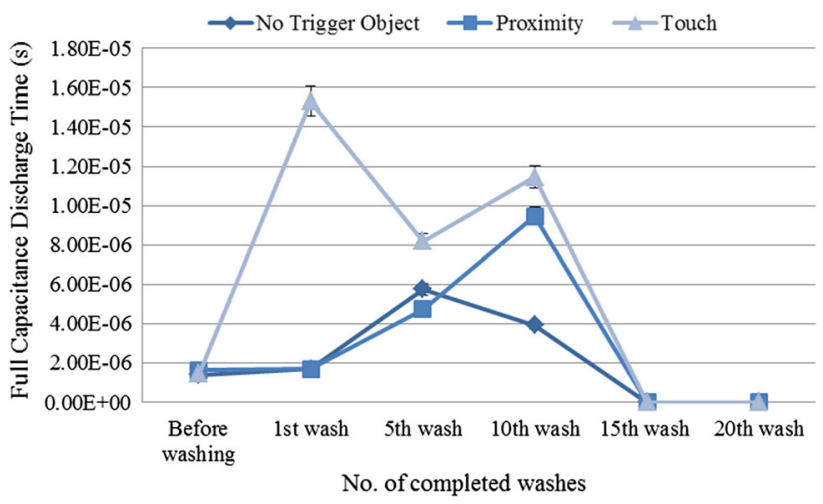

Fig. 31 Graph comparing fall time (full discharging time) of e-textile 4 capacitive circuit when there is no detection, proximity detection, and touch detection of a human hand after multiple washing cycles 


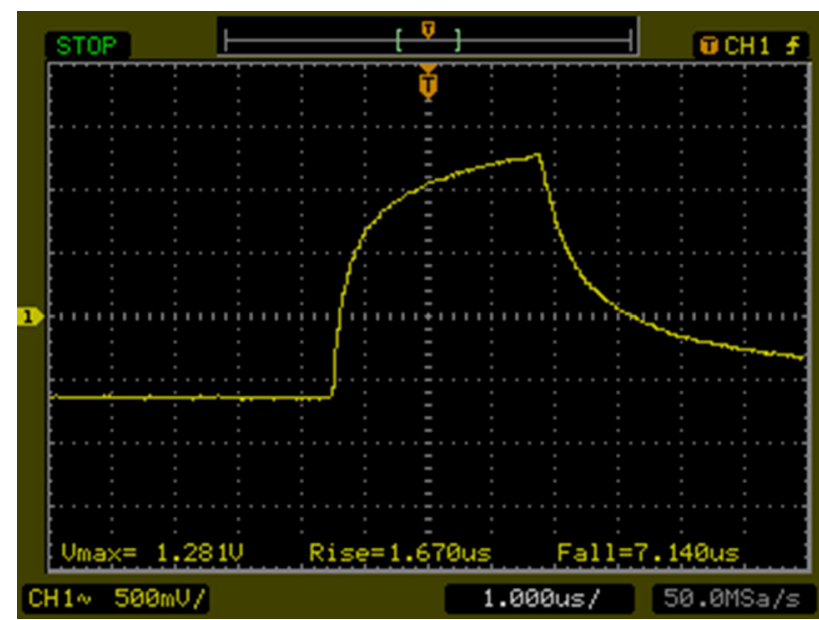

Fig. 32 Example oscilloscope waveform of e-textile 4 after ten washes with no trigger object detection

On Fig. 31, touch detection is even more distinguishable by the proximity chip compared to proximity detection. Nonetheless, this ability for the proximity chip to detect touch and proximity declines with successive washing cycles until it stops functioning which is shown through the general negative correlation trend lines for touch and proximity.

In comparison, for e-textile 4's first wash the rise time touch detection value increases from the before washing value by $3 \%$ and on the tenth wash $478 \%$. Compare this to touch, whereby for the first wash the percentage changes from before washing is $949 \%$ and on the tenth wash becomes $684 \%$. Although the percentage change seems very high, this is actually a positive indicator at how successful the circuit is at detecting a trigger object compared to when there is no trigger object detected.

An important observation from this experiment is the ability to predict when the e-textile would stop-functioning when analyzing the collected rise and fall time data. Agreeing with the experimental hypothesis and RC curve theory, as the human hand gets closer to the copper sensing electrode the rise time and fall time increases whilst the charging voltage $\left(V_{\max }\right)$ decreases. At the tenth wash, the fall and rise times for proximity and touch sensing appear to be similar before it fails. This observation appears to hold for all e-textiles under test and statistically supported by looking at percentage difference. Examining the data for Figs. 28 and 30, e-textile 3 has a $17.7 \%$ percentage difference between proximity and touch rise times after the first wash compared to an $8.6 \%$ percentage difference after the tenth wash. Whilst e-textile 4 has a $69.2 \%$ percentage difference between proximity and touch rise times after the first wash compared to a $15.3 \%$ percentage difference after the tenth wash. Consequently, experimental results provide an indication the e-textile is likely to fail when the difference between rise and fall times for proximity and touch detection becomes its smallest. This is an interesting result, as when this behavior is observable it would suggest the e-textile circuit will not survive and hence no longer have functionality if subsequently washed. This behavior is also indicated by the RC curve waveform captured by the oscilloscope.

The detrimental washing cycle effect on the e-textiles' functionality is clear when comparing the RC waveforms of an e-textile that is unwashed to one washed ten times. Comparing Figs. 12 with Figs. 33, 34 and 35, the latter have a more distinct sawtooth waveform shape. The RC waveforms for the circuits within the washed e-textiles have their capacitor's transient response ending prematurely and not appearing to reach a steady state response before the capacitor discharges.

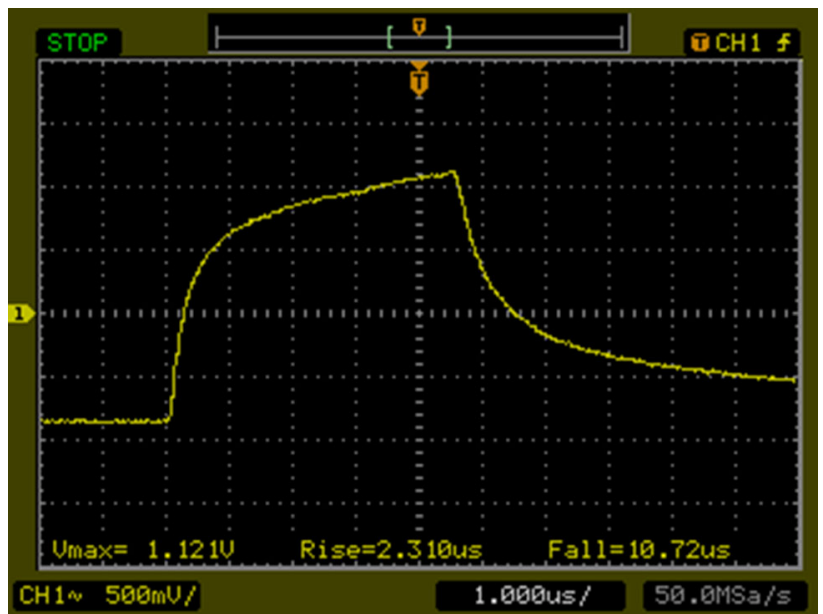

Fig. 33 Example oscilloscope waveform of e-textile 4 after ten washes with proximity detection of a human hand

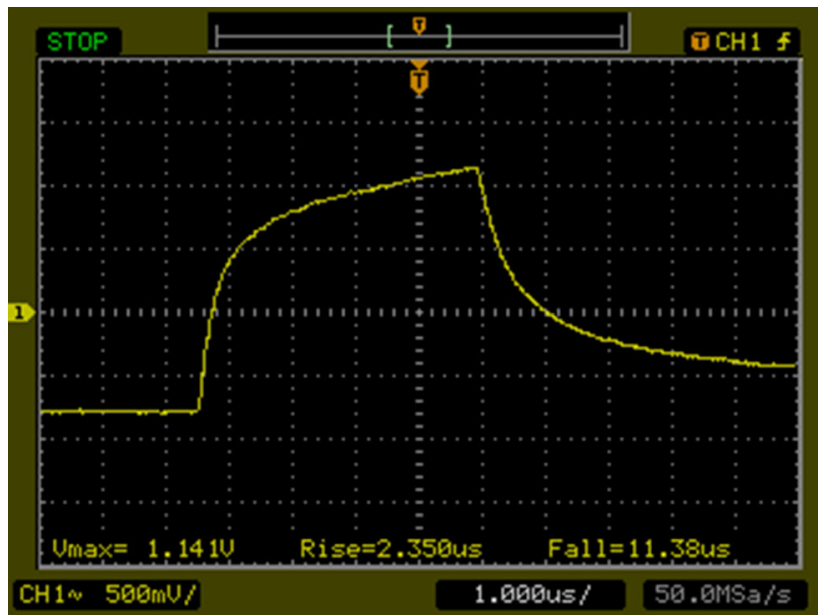

Fig. 34 Example oscilloscope waveform of e-textile 4 after ten washes with touch detection of a human hand 


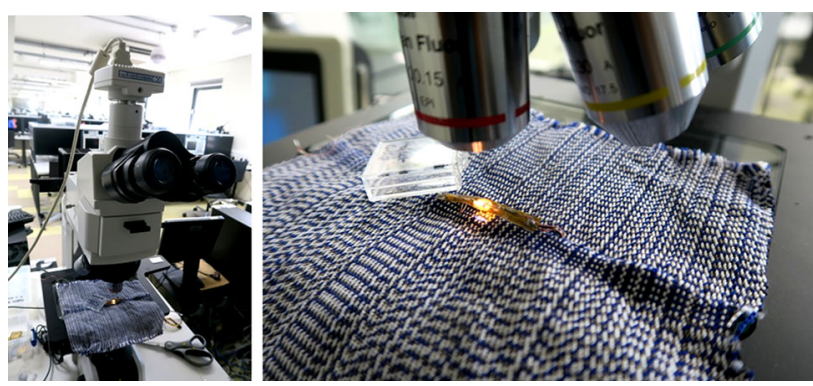

Fig. 35 Microscope analysis of PDMS-packaged capacitive circuit integrated into a textile after consumer washing machine experiment

\subsection{Microscopic inspection of washed e-textile circuit hydrophobic packaging}

Microscopic images were taken of extracted circuits from washed e-textiles to discover how mechanical abrasions of the washing cycle affected the PDMS hydrophobic layer. The failure of the circuits in the washing test could have also been due to bending and twisting of the circuit during the washing test.

E-textile circuits no longer functioned when wires were pulled from the solder joint connected to the circuit. When this occurred, readings could no longer be recorded from the circuit. This occurred for e-textile 2, 3, and e-textile 4 . This strain upon the wires could be caused by the circuit being bent, twisted, and its wires becoming caught in-between folds of the other textiles. It is likely that aqueous solution entered the packaging once a resulting tear in the PDMS occurred, as the wires on the circuit detached through the PDMS conformal and hydrophobic layer, reaching the components to cause failure. The microscope was used to investigate this hypothesis.

This presents an improvement to this e-textile system, to have the wires fully integrated into the textile so they are not detached during the wash (Fig. 36).

A Nikon EVB-100 microscope (Fig. 35) was used to inspect the PDMS conformal layer of the circuits embedded into the e-textile after washing. They were extracted through the e-textile by irreversibly cutting through the

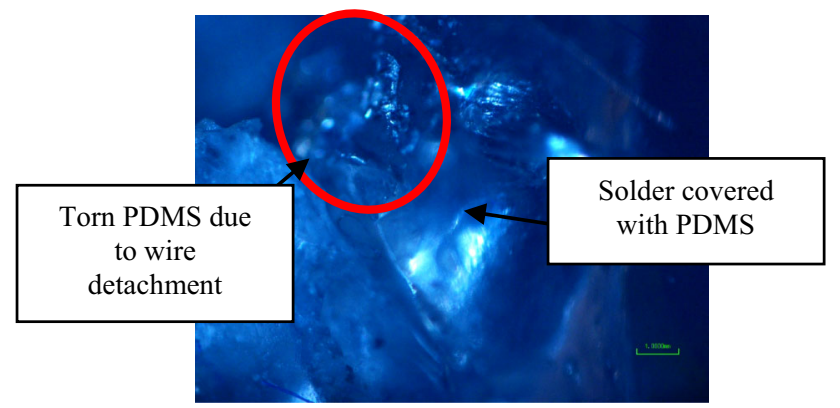

Fig. 36 Microscopic image of torn PDMS conformal coating due to wire being pulled from joint during washing

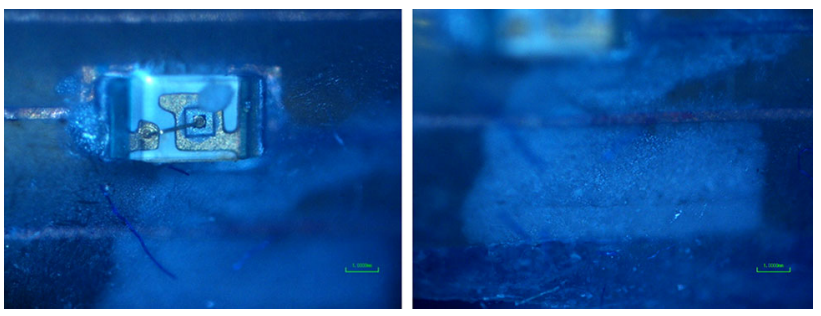

Fig. 37 Moisture surrounding surface mount component after wash test due to tear in conformal PDMS packaging
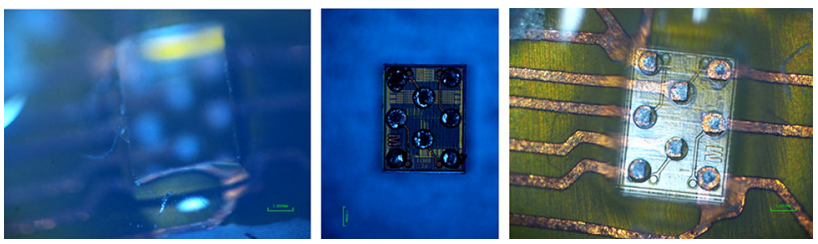

Fig. 38 Microscopic image of conformal PDMS retaining shape despite detachecd central chip (left) image of uneven thermally cured solder bumps underneath chip (middle) and image of embossed PDMS on circuit following chip detachment (right)
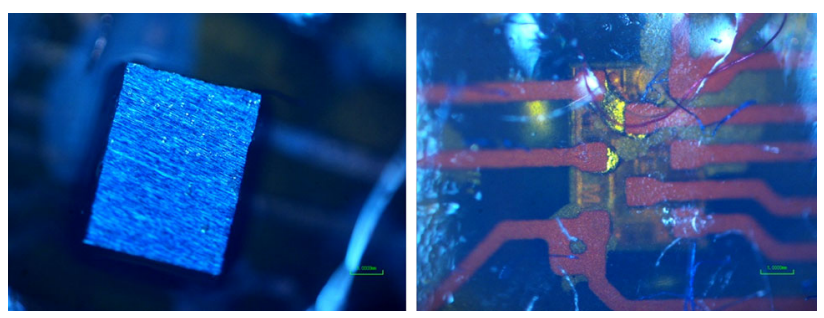

Fig. 39 Microscopic images of conformal PDMS layer on capacitive sensing chip still on circuit after $400 \mathrm{rpm}$ washing spin speed (left) and view underneath circuit to see chip connection to solder pads (right)

textile, as the circuits were completely integrated into a textile using industrial textile contruction machinery. Microscopic analysis revealed that wires pulled out of the solder joint during washing created an opening (Fig. 36) in the packaging causing water to reach the components.

Evidence of water reaching the components was confirmed by moisture regions surrounding components underneath the PDMS conformal layer (Fig. 37).

To prevent this, the wires could be integrated within the textile as opposed to exiting the channel where the circuit is located.

Furthermore, the $800 \mathrm{rpm}$ spin speed used for washing-textile 1 and 2 caused the central capacitive sensing chip to detach from the solder pads on the circuit (Fig. 38, left). Underneath the chip (Fig. 38, middle) it shows that all solder bumps of the PCF8883US have thermally cured unevenly. This contributed to its detachment due to reduced mechanical adhesion. There is also evidence of PDMS acting as an elastomeric underfill for the chip, as the 
bottom of the chip has embossed the PDMS underneath it (Fig. 38, right).

However, Fig. 39 shows that for a reduced washing spin speed of $400 \mathrm{rpm}$ the central PCF8883US IC chip still adhered onto the circuit whilst surviving a greater number of cycles. PDMS' robustness to protect the SMDs on this flexible circuit is similar to results in literature (Vervust et al. 2012), which reported PDMS-encapsulated electronics being able to withstand domestic and industial textile washing.

This microscopic result further supports the use of a reduced washing spin speed of $400 \mathrm{rpm}$, to ensure that all surface mount components adhere to the circuit. However, a circuit packaging which is more resistant to tearing is needed to prevent water, detergent, and fabric conditioner solution entering the circuit and causing malfunction. Given the benefits of PDMS shown in this paper, this can be explored by having a PDMS that is more rigid at the wire solder joints or another substrate to ensure wires are secured onto the circuit. However, it has also revealed that improved soldering of the wires and potentially a need for underfill on the components are required for greater durability.

\section{Conclusions}

This paper explored the relationship between PDMS crosslinking and its hydrophobicity and permeability to aqueous solvent solutions specific to textile washing. Results showed that changing the amount of cross-linking can control the hydrophobicity of the PDMS to water, detergent, and fabric conditioner solutions. Furthermore, the Sylgard 184 standard 10:1 mixing ratio for electrical/electronic applications is not optimal for washable electronics integrated into textiles as it is not tailored to hydrophobicity to water, detergent, and fabric conditioner commonly used in consumer washing of textiles. Therefore, for PDMS to be used as a hydrophobic microsystem packaging layer for textile integration and survive washing, a mixture with lower cross-linking such as 20:1 should be used. Having 20:1 instead of 10:1 PDMS and the permeability of water can reduce by approximately $45-48 \%$. The reduced degree of swelling with time behavior holds for detergent (DetergentC) but is overall inconclusive with fabric conditioner (FabricCondC) as the solution discourages permeation; so this therefore requires further investigation. Other factors such as temperature and PDMS dimensions are influential (Varnaite and Katunskis 2009).

Furthermore, washing tests with an average $40.3 \mu \mathrm{m}$ thick conformal 20:1 PDMS flexible sensory circuits were completed using the ISO 6330:2012 standard at different temperatures and durations. This was to evaluate PDMS' machine-washing durability when circuits are integrated into a textile.

The washing test revealed that washing cycle rotary spin speed has a strong influence on the lifetime of the circuits. With an $800 \mathrm{rpm}$ washing spin speed at $30{ }^{\circ} \mathrm{C}$ for $15 \mathrm{~min}$, all circuits examined failed after one wash with detergent and fabric conditioner included. Therefore, the washing spin speed was reduced to its lowest setting at $400 \mathrm{rpm}$ for two other washing speeds/cycle which survived between 10 and 15 cycles. The silk-encapsulated multi-strand copper wire used in the washing test snapped due to mechanical abrasions of the washing machine. $2 \mathrm{~kg}$ of cotton textile was included in the wash, which resulted in the circuits being folded, bent, and twisted during the washing cycle. This caused circuits to have their wires pulled from their solder joints. Subsequent washing with the torn hydrophobic PDMS packaging allowed aqueous washing solution to reach the components and caused the circuit to become non-functioning.

Comparing the results from this paper to that in literature (Linz et al. 2005; Merritt et al. 2009; Varnaite and Katunskis 2009), not all of these literature test the functionality of a circuit with embedded electronic components. Linz et al. (2005) was most similar to this work, as it had a transponder electronic module but encapsulated with globtop and rigid packaging moulding. Yet, Merritt et al. (2009) was passive sensing electrodes, and Varnaite et al. (2009) yarns acting as conductive wires respectively.

The work reported in this paper presents washing test data specific to advanced e-textiles, whereby the electronics are fully integrated into the textile, achieving double the number of washes compared to other techniques. Literature that has reported high numbers of washing cycle survival such as 20 (Kazani et al. 2012) and 50 (Kaappa et al. 2017) washing cycles did not feature electronic circuits in the washing machine but only printed conductive tracks and dry electrodes respectively. This paper therefore contributes to the state of the art knowledge for washing cycle durability and robustness for e-textiles; where wires and electronic circuits are packaged and integrated into a textile for washing.

As future work, mechanical experiments will be conducted on the 20:1, average $40.3 \mu \mathrm{m}$-thick PDMS encapsulated sensory circuits to identify which kind of mechanical deformation caused the greatest circuit damage during machine washing. Hence, cyclic twisting and cyclic bending tests will be performed on these circuits to test their durability, with anticipation that causes of consequent failure will be similar to those in the washing test. Further washing tests investigating the effect of washing temperature on e-textile sursival would help towards creating new e-textile washing standards for commercial usage. 
Acknowledgements This research was funded in the UK by the Engineering and Physical Sciences Research Council (EPSRC) via the project: Novel manufacturing methods for Functional Electronic TexTiles (FETT). Grant number: EP/M015149/1. This research acknowledges the work of Helga Nunes-Matos for her contribution in creating the woven e-textile demonstor experimented on in this paper.

Open Access This article is distributed under the terms of the Creative Commons Attribution 4.0 International License (http://creative commons.org/licenses/by/4.0/), which permits unrestricted use, distribution, and reproduction in any medium, provided you give appropriate credit to the original author(s) and the source, provide a link to the Creative Commons license, and indicate if changes were made.

\section{References}

Armani D, Liu C, Aluru N (1999) Re-configurable fluid circuits by PDMS elastomer micromachining. In: Technical Digest. IEEE International MEMS 99 Conference. Twelfth IEEE International Conference on Micro Electro Mechanical Systems (Cat. No. 99CH36291). IEEE, pp 222-227

BOSCH Customer Support. From Bosch: https://www.bosch-home. co.uk/customer-service/get-support/which-washing-machine-pro gramme-should-i-use. Accessed July 2018

Brook MA, Organic O (2000) Polymer Chemistry. Wiley, New York

Brooks JH, Das UK, Smith JL (1989) Effect of lubrication on tensile, frictional, and weaving properties of sirospun wool yarn. Text Res J 59(7):382-388

Cherenack K, Zysset C, Kinkeldei T, Münzenrieder N, Tröster G (2010) Woven electronic fibers with sensing and display functions for smart textiles. Adv Mater 22(45):5178-5182

Essö C (2007). Modifying Polydimethylsiloxane (PDMS) surfaces

FETT Project Homepage (2011). From FETT Project: www.fett.ecs. soton.ac.uk. Accessed Oct 2015

Frank S, Kuijper A (2017) Authenticap-A touchless vehicle authentication and personalization system. In: European Conference on Ambient Intelligence. Springer, Cham, pp 46-63

Fu YY, Chan YL, Yang MH, Chan YC, Virkki J, Björninen T, Ukkonen L (2015) Experimental study on the washing durability of electro-textile UHF RFID tags. IEEE Antennas Wirel Propag Lett 14:466-469

Fujii T (2002) PDMS-based microfluidic devices for biomedical applications. Microelectron Eng 61:907-914

George B, Zangl H, Bretterklieber T, Brasseur G (2010) A combined inductive-capacitive proximity sensor for seat occupancy detection. IEEE Trans Instrum Meas 59(5):1463-1470

Honda T, Miyazaki M, Nakamura H, Maeda H (2005) Controllable polymerization of $\mathrm{N}$-carboxy anhydrides in a microreaction system. Lab Chip 5(8):812-818

Jagiella M, Fericean S, Dorneich A (2006) Progress and recent realizations of miniaturized inductive proximity sensors for automation. IEEE Sens J 6(6):1734-1741

Jeong JW, McCall JG, Shin G, Zhang Y, Al-Hasani R, Kim M, Hong DY (2015) Wireless optofluidic systems for programmable in vivo pharmacology and optogenetics. Cell 162(3):662-674

Kaappa ES, Joutsen A, Cömert A, Vanhala J (2017) The electrical impedance measurements of dry electrode materials for the ECG measuring after repeated washing. Res J Text Appar 21(1):59-71

Kang TJ, Kim MS (2001) Effects of silicone treatments on the dimensional properties of wool fabric. Text Res J 71(4):295-300

Kazani I, Hertleer C, De Mey G, Schwarz A, Guxho G, Van Langenhove L (2012) Electrical conductive textiles obtained by screen printing. Fibres Text East Eur 20(1):57-63
Kim HT, Jeong OC (2011) PDMS surface modification using atmospheric pressure plasma. Microelectron Eng 88(8):2281-2285

Lee JN, Park C, Whitesides GM (2003) Solvent compatibility of poly (dimethylsiloxane)-based microfluidic devices. Anal Chem 75(23):6544-6554

Linz T, Kallmayer C, Aschenbrenner R, Reichl H (2005) Embroidering electrical interconnects with conductive yarn for the integration of flexible electronic modules into fabric. In: Wearable computers, 2005. Proceedings. Ninth IEEE International Symposium on, IEEE, pp 86-89

Mark JE, Pan SJ (1982) Reinforcement of polydimethylsiloxane networks by in situ precipitation of silica: a new method for preparation of filled elastomers. Macromol Rapid Commun 3(10):681-685

Mattmann C, Clemens F, Tröster G (2008) Sensor for measuring strain in textile. Sensors 8(6):3719-3732

Merck MillioPore Homepage (2018), from Merck Millipore: http:// www.merckmillipore.com/GB/en/product/Q-POD-Ele ment,MM_NF-C84730. Accessed July 2018

Merritt CR, Nagle HT, Grant E (2009) Fabric-based active electrode design and fabrication for health monitoring clothing. IEEE Trans Inf Technol Biomed 13(2):274-280

Morent R, De Geyter N, Axisa F, De Smet N, Gengembre L, De Leersnyder E, Leys C, Vanfleteren J, Rymarczyk-Machal M, Schacht E, Payen E (2007) Adhesion enhancement by a dielectric barrier discharge of PDMS used for flexible and stretchable electronics. J Phys D Appl Phys 40(23):7392-7401

Ojuroye OO, Torah RN, Komolafe AO, Beeby SP (2019) Embedded capacitive proximity and touch sensing flexible circuit system for electronic textile and wearable systems. IEEE Sensors Journal

Palchesko RN, Zhang L, Sun Y, Feinberg AW (2012) Development of polydimethylsiloxane substrates with tunable elastic modulus to study cell mechanobiology in muscle and nerve. PLoS One 7(12): 51499

Rantanen J, Impiö J, Karinsalo T, Malmivaara M, Reho A, Tasanen M, Vanhala J (2002) Smart clothing prototype for the arctic environment. Pers Ubiquit Comput 6(1):3-16

Ren X, Lu H, Zhou JG, Chong PLG, Yuan W, Noh M (2017) Porous polydimethylsiloxane as a gas-liquid interface for microfluidic applications. J Microelectromech Syst 26(1):120-126

Rus S, Sahbaz M, Braun A, Kuijper A (2015) Design factors for flexible capacitive sensors in ambient intelligence. In: European Conference on Ambient Intelligence. Springer, Cham, pp 77-92

Satharasinghe A, Hughes-Riley T, Dias T (2018) Photodiodes embedded within electronic textiles. Sci Rep 8(1):16205

Washing TD (2000) Drying procedures for textile testing. ISO 6330-2000

Tao X, Huang TH, Shen CL, Ko YC, Jou GT, Koncar V (2018) Bluetooth Low Energy-Based Washable Wearable Activity Motion and Electrocardiogram Textronic Monitoring and Communicating System. Adv Mater Technol 3(10):1700309

Toepke MW, Beebe JD (2006) PDMS absorption of small molecules and consequences in microfluidic applications. Lab Chip 6(12):1484-1486

van Dam RM (2006) Solvent-resistant elastomeric microfluidic devices and applications. Doctoral dissertation, California Institute of Technology

Varnaite S, Katunskis J (2009) Influence of washing on the electric charge decay of fabrics with conductive yarns. Fibres Text East Eur 17(5):69-75

Vervust T, Buyle G, Bossuyt F, Vanfleteren J (2012) Integration of stretchable and washable electronic modules for smart textile applications. J Text Inst 103(10):1127-1138 
Wainwright HL (2016) Design, evaluation, and applications of electronic textiles. In: Performance Testing of Textiles. Woodhead Publishing, pp 193-213

Wang EJ, Garrison J, Whitmire E, Goel M, Patel S (2017) Carpacio: repurposing capacitive sensors to distinguish driver and passenger touches on in-vehicle screens. In Proceedings of the 30th Annual ACM Symposium on User Interface Software and Technology, ACM, pp 49-55
Wei H, Chueh BH, Wu H, Hall EW, Li CW, Schirhagl R, Zare RN (2011) Particle sorting using a porous membrane in a microfluidic device. Lab Chip 11(2):238-245

Zysset C, Kinkeldei TW, Munzenrieder N, Cherenack K, Troster G (2012) Integration method for electronics in woven textiles. IEEE Trans Compon Packag Manuf Technol 2(7):1107-1117

Publisher's Note Springer Nature remains neutral with regard to jurisdictional claims in published maps and institutional affiliations. 